\title{
AN OVERVIEW OF GRAVITATIONAL-WAVE SOURCES
}

\author{
CURT CUTLER \\ Max Planck Institute for Gravitational Physics, 14476 Golm, Germany \\ KIP S. THORNE \\ Theoretical Astrophysics, California Institute of Technology, Pasadena, CA 91125 \\ in consultation with \\ LARS BILDSTEN, ALESSANDRA BUONANNO, CRAIG HOGAN, \\ VASSILIKI KALOGERA, BENJAMIN J. OWEN, E. STERL PHINNEY, \\ THOMAS A. PRINCE, FREDERIC A. RASIO, STUART L. SHAPIRO, \\ KENNETH A. STRAIN, GREG USHOMIRSKY AND ROBERT V. WAGONER. \\ We review current best estimates of the strength and detectability of the gravi- \\ tational waves from a variety of sources, for both ground-based and space-based \\ detectors, and we describe the information carried by the waves.
}

\section{Preamble}

Major relativity conferences such as GR16 traditionally include an overview talk on gravitationalwave (GW) sources. Some exfellent recent ones include those by Flanagan $\mathrm{Z}$, Finn $\mathrm{Z}$, and Bender et al. $\mathrm{B}$. Such talks are always by theorists, and can be described as basically informed speculation, since until now detectors sufficiently sensitive to detect GW's have not existed. But km-scale laser interferometers (IFO's) are now coming on-line, and it seems very likely these will detect mergers of compact binaries within the next seven years, and possibly much sooner. There are several other classes of sources that the ground-based IFO's might well detect: massive star collapse (supernovae and hypernovae), rapidly rotating neutron stars, and possibly a GW stochastic background created in the early universe. A space-based interferometer, LISA, is also planned (though not yet fully funded), and could fly in $\sim 2011$. There is one type of source - short-period galactic binaries - that LISA is guaranteed to observe (at its planned sensitivity), plus a list of very promising candidates: the inspiral and merger of supermassive black holes (SMBH's), the inspiral and capture of compact objects by SMBH's, and sources in the very early universe.

In this article we review the various GW sources that have been studied, for both ground and space-based detectors, summarizing the best available estimates for rates and source strengths and describing some of the informa-

cutlerThorne: submitted to World Scientific on February 5, 2008 
tion that may be extracted from the waves. We will try to emphasize what is new, but much here is not, and indeed many sections of this paper have been adapted, with minor updating, from an earlier review by Thorne 6 or from a publicly available LIGO-Project document by Thorne 5 (based on extensive input from the colleagues listed as consultants at the beginning of this article).

Gravitational-wave detection efforts focus on four frequency bands: (i) The extremely low frequency (ELF) band, $10^{-15}$ to $10^{-18} \mathrm{~Hz}$, in which waves will be sought via their imprint on the polarization of the cosmic microwave background $(\mathrm{CMB})$ radiation $\mathbf{6}$, and the only expected source is primordial gravitational fluctuations amplified by inflation of the universe. (ii) The very low frequency (VLF) band, $10^{-7}$ to $10^{-9} \mathrm{~Hz}$, in which the waves are sought using high-stability pulsar timing $\mathrm{G}$, and the expected sources are processes in the very early universe and extremely massive binary black hole systems. (iii) The low frequency (LF) band, $10^{-4}$ to $1 \mathrm{~Hz}$, in which LISA will operate, and (iv) the high frequency (HF) band, 1 to $10^{4} \mathrm{~Hz}$, in which earth-based detectors (interferometers and resonant-mass detectors) operate.

In this article we shall confine attention to the LF and HF bands, since that is where the richest variety of sources reside, that is where the vast majority of the current experimental activity concentrates, and that is where the first firm detections are likely to be made. We shall not say much about details or current status or plans for gravitational wave detection, as there are mapy other review articles devoted to this e.g. those by Kawamura Schutz 0 , Robertson 10 , and Barish and Weiss 11.

In Sec. 2 we shall discuss high-frequency sources being sought by earthbased detectors, and in Sec. 3, low-frequency sources that will be sought by LISA (and some of which are already being sought by Doppler tracking of spacecraft 12$)$.

\section{High-Frequency Sources Sought by Earth-Based Detectors}

\subsection{Fiducial Noise Curves}

In discussing gravitational-wave sources, it is important to put them in the context of detector sensitivities, both near-term and planned. As is discussed in Kawamura's chapter in this volume 8 , the first-generation kilometer-scale interferometric detectors (LIGO, VIRGO, GEO600, TAMA300) were being assembled at the time of GR16 and will begin their initial GW searches about the time that our review is published. In about 2007 these first-generation interferometers (IFOs) will be upgraded to produce second-generation IFOs, with noise curves that can be adjusted by adjusting the position and reflec-

cutlerThorne: submitted to World Scientific on February 5, 2008 
tivity of one mirror. In parallel with this IFO experimental effort, a netwerk of narrow-band resonant-mass detectors is operating and being improved 13 .

In this review we shall find it convenient to evaluate prospects for detection with respect to three representative, fiducial noise curves: (i) that for the first-generation LIGO IFOs (the LIGO-I noise curve); (ii) that for the second-generation LIGO IFOs (sometimes called advanced LIGO IFOs and sometimes called LIGO-II IFOs), when they are operated in a "wide-band" mode (the WB LIGO-II noise curve); and (iii) that for second-generation IFOs operated in a "narrow-band" mode that is optimized for sources near $600 \mathrm{~Hz}$ frequency (the NB LIGO-II noise curve). Though focusing on LIGO smacks of American egocentrism, the reason is pragmatic: the first-generation VIRGO noise curve would largely lie between ithe initial LIGO (LIGO-I) curve and the advanced LIGO (LIGO-II) curves anyway, VIRGO does not publish an official second-generation noise curve, and GEO600 and TAMA300 are purely first-generation instruments.

Our three fiducial noise curves are shown in Fig. 1 along with the wave strengths for a number of HF sources to be discussed below. The horizontal axis in the figure is GW frequency $f$; the vertical axis is the square root of the spectral density of an IFO's arm-length difference, i.e. the IFO's "strain per root Hertz" $\tilde{h}(f) \equiv \sqrt{S_{\Delta L / L}(f)}$. The (dimensionless) rms noise in a bandwidth $\Delta f$ around frequency $f$ is given by $h_{\mathrm{rms}}=\tilde{h}(f) \sqrt{\Delta f}$.

Notice that, compared to LIGO I, the WB LIGO-II IFOs (i) will lower the amplitude noise by a factor $\sim 15$ at the frequencies of best sensitivity $f \sim 100$ $200 \mathrm{~Hz}$, and (ii) will widen the band of high sensitivity at both low frequencies (pushing it down to $\sim 20 \mathrm{~Hz}$ ) and high frequencies (pushing it up to $\sim 1000$ $\mathrm{Hz}$ ). The lowered WB noise at optimal frequencies will increase the event rate for distant, extragalactic sources by a factor $\sim 15^{3} \simeq 3000$. Opening up lower and higher frequency bands will bring us into the domains of new sources: colliding, massive black holes and stochastic background at low frequencies; low-mass X-ray binaries, fast pulsars and tidal disruption of neutron stars by black holes at high frequencies. Noise-curve reshaping, as illustrated by the NB LIGO-II IFO, will reduce the noise by a factor $\sim 3$ to 5 relative to the WB IFO within some chosen narrow frequency band $\Delta f / f \sim 0.2$ in which targeted periodic sources (e.g. low-mass X-ray binaries) are expected to lie. We shall refer to this as "narrow-band tuning" of the IFO.

The expected LIGO II noise curves depend somewhat on whether the mirrors' substrate is made from sapphire (the preferred material) or from fused silica (the backup). We assume sapphire in this article. If problems with sapphire force fused silica to be used, the WB IFOs will likely be a few tens of percent more noisy than Fig. 11 near the noise-curve minimum, and 


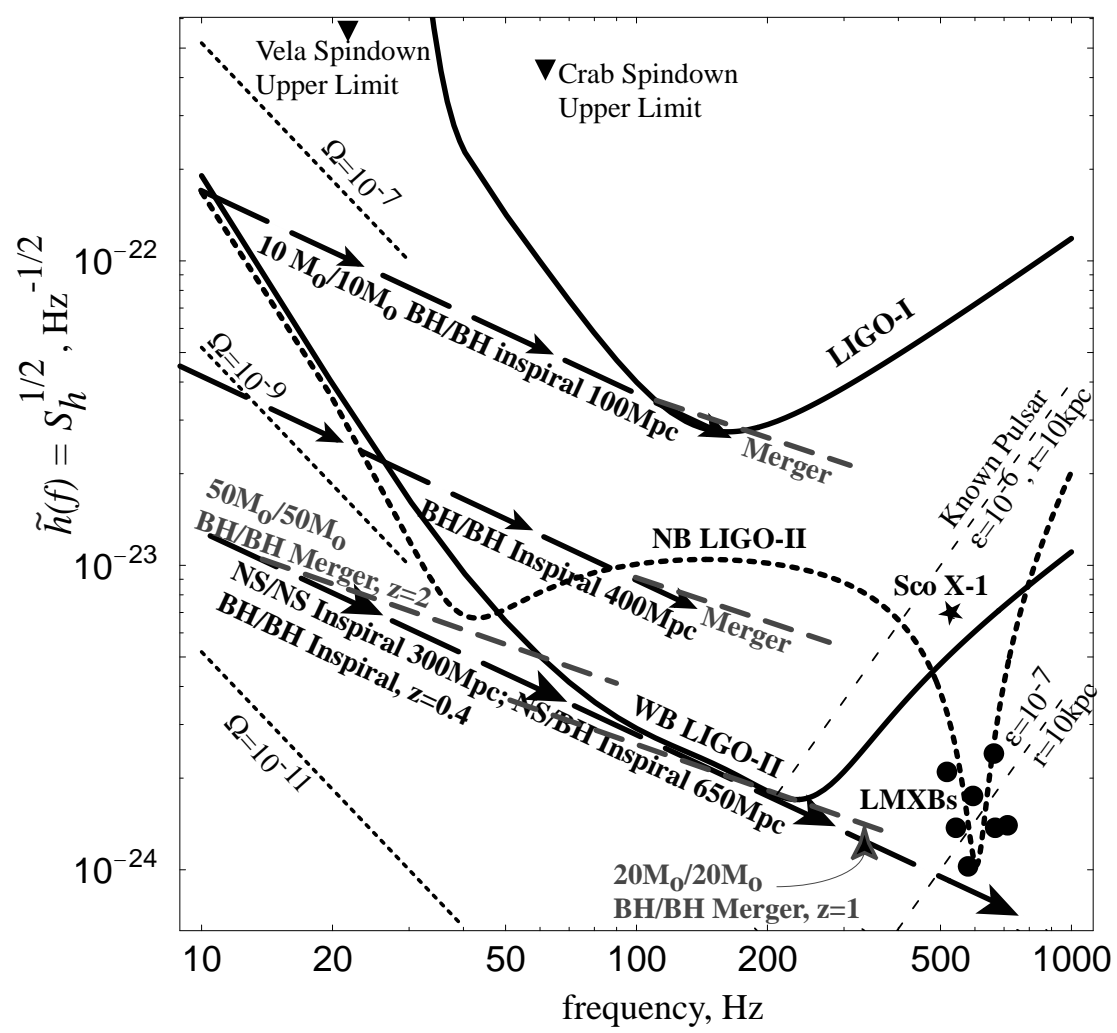

Figure 1. The noise $\tilde{h}(f)$ in several planned LIGO interferometers plotted as a function of gravity-wave frequency $f$, and compared with the estimated signal strengths $\tilde{h}_{s}(f)$ from various sources. The signal strength $\tilde{h}_{s}(f)$ is defined in such a way that, wherever a signal point or curve lies above the interferometer's noise curve, the signal, coming from a random direction on the sky and with a random orientation, is detectable with a false alarm probability of less than one per cent; see the text for greater detail and discussion.

the overall GW event rate may be worse by about a factor 2 .

Figure 1 shows, along with the noise curves, the estimated signal strengths $\tilde{h}_{s}(f)$ for various sources. These signal strengths are defined in such a way 14 that the ratio $\tilde{h}_{s}(f) / \tilde{h}(f)$ is equal to the ratio of signal $S$ to noise threshold $T$, rms averaged over source directions and orientations, $\tilde{h}_{s}(f) / \tilde{h}(f)=$ $\left\langle S^{2} / T^{2}\right\rangle^{1 / 2}$, with the threshold being that at which the false alarm probability is one per cent when using the best currently known, practical data analysis 
algorithm. (For broad-band sources, the algorithm is assumed to integrate over a bandwidth equal to frequency and to use the output from LIGO's two $4 \mathrm{~km}$ IFOs and one $2 \mathrm{~km}$ IFO 15, thereby removing all non-Gaussian noise. For periodic sources such as spinning neutron stars, the algorithm uses data from only one $4 \mathrm{~km}$ IFO, usually narrow banded, the noise again is assumed Gaussian, and the signal is integrated for $10^{7} \mathrm{sec}$, except in cases such as Low Mass X-Ray Binaries where there is little gain from integrating so long.) This definition of $\tilde{h}_{s}(f)$ means that, wherever a signal point or signal curve lies above the IFO noise curve, the signal, coming from a random direction on the sky and with a random orientation, is detectable with a false alarm probability of less than one per cent.

\subsection{Overview of High-Frequency Sources}

In this section, we shall discuss the high-frequency sources briefly, in turn, and then in subsequent sections we shall discuss them in greater detail, focusing on the likelihood of detection and the science we expect to extract from detected waves.

The three arrowed, long-dashed lines in Fig. 1 represent the signal $\tilde{h}_{s}(f)$ from neutron-star (NS) and black-hole (BH) binaries in the last few minutes of their inspiral, assuming masses $M=1.4 M_{\odot}$ for each NS and $M=10 M_{\odot}$ for each $\mathrm{BH}$. These sources are best searched for by the method of matched filters 16. Using matched filters, LIGO's initial IFOs can detect NS/NS inspirals (with a 1 per cent false alarm probability) out to a distance of $20 \mathrm{Mpc}$ [top arrowed line]; the wide-band advanced (LIGO-II) IFOs can do so 15 times farther, out to $300 \mathrm{Mpc}$ for NS/NS and out to $650 \mathrm{Mpc}$ for NS/BH [bottom arrowed line]. For BH/BH inspiral, the wide-band IFOs can see so far that cosmological effects are important. For definiteness, throughout this proposal we assume a Hubble expansion rate $H_{o}=65 \mathrm{~km} / \mathrm{s} / \mathrm{Mpc}$, a coldmatter density 0.4 of that required to close the universe $\Omega_{M}=0.4$, and a vacuum energy density (cosmological constant) 0.6 of closure $\Omega_{\Lambda}=0.6$. Then the wide-band IFOs can see $\left(10 M_{\odot} / 10 M_{\odot}\right) \mathrm{BH} / \mathrm{BH}$ inspirals out to a cosmological redshift $z=0.4$. The binary inspiral rates at these advanced IFO distances are likely to be many per year; see Box 1 at the end of this section. The middle arrowed line is the signal from $\mathrm{BH} / \mathrm{BH}$ inspiral at $400 \mathrm{Mpc}$, where the geometric mean of the event-rate estimates for $\mathrm{BH} / \mathrm{BH}$ field binaries is three per year (third column of table in Box 1).

The tidal disruption of a NS by its $\mathbf{B H}$ companion at the endpoint of NS/BH inspiral should produce gravitational waves that carry detailed information about the NS structure and equation of state. The advanced IFOs

cutlerThorne: submitted to World Scientific on February 5, 2008 
may detect these waves and extract their information; see Sec. 2.4. This tidal disruption is a promising candidate for the trigger of some gamma-ray bursts, as is the final merger of the two NS's in a NS/NS binary. A gammaburst / gravitational-wave coincidence would be of great value in revealing the nature of gamma-burst sources; see Sec. 2.8

For $\mathrm{BH} / \mathrm{BH}$ binaries much heavier than $\sim 10 M_{\odot} / 10 M_{\odot}$, most of the gravitational signal is likely to come from the black holes' merger and the vibrational ringdown of the final black hole, rather than from the inspiral. Rough estimates discussed in Sec. 2.5 suggest that, if the holes are rapidly spinning (within a few per cent of the fastest spin allowed, i.e. $a / m \gtrsim 0.98)$, then the wide-band IFOs can see the merger waves from two $20 M_{\odot}$ holes out to $z=1$ and two $50 M_{\odot}$ holes out to $z=2$; see the downsloping, non-arrowed, dashed lines in Fig. 1. The event rates at these distances could well be many per year, and the waves from such mergers will carry rich physical and astrophysical information; see Sec. 2.5.

The triangles, star, large dots, and up-sloaping short-dashed lines in Fig. 1 represent signals from slightly deformed, spinning neutron stars. The most interesting of these is a class of objects called low-mass X-ray binaries (LMXB's). These are neutron stars that are being torqued by accretion from a companion, but that seem to be locked into spin periods in the range $\sim 300-600$ revolutions per second. The most plausible explanation for this apparent locking is that the accretion is producing an asymmetry that radiates gravitational waves, which torque the star's spin down at the same rate as accretion torques it up 17.2 . Assuming this to be true, one can deduce an LMXB's wave strength $\tilde{h}_{s}$ from its measured X-ray flux and its spin frequency [with the frequency inferred, sometimes to within a few $\mathrm{Hz}$ but not better, from nearly coherent oscillations (NCOs) in type-I X-ray bursts, or less reliably from frequency splittings of quasiperiodic (QPO) X-ray oscillations 19 .] The spin frequency, and thence the gravitational-wave frequency, will wander somewhat due to fluctuations in accretion (which can be estimated by monitoring the X-ray flux) and due to poorly known orbital parameters. As a result, in searching for an LMXB's waves one can only perform coherent integrations for about 20 days; thereafter, one must stack the signals incoherently, allowing for unknown shifts of the wave frequency 20121. When one uses this "stack-slide" method of data analysis, the resulting signal strengths may improve only slightly for integration times longer than 20 days 20 . (The most sensitive LMXB searches will probably be with NB IFO's, with the optimum sensitivity band re-adjusted every $\sim 20$ days to cover the whole range $\sim 400-1000 \mathrm{~Hz}$.)

Assuming 20 days of integration using a single 4-km IFO, the estimated

cutlerThorne: submitted to World Scientific on February 5, 2008 
signal strengths and frequencies for the strongest known LMXB's are shown by the big dots and the star in Fig. 1. The estimated strengths assume a steady-state balance of accretion torque by gravitational-wave torque, and that the GW's are generated by density inhomogneneities (as opmosed to NS wobble or unstable modes), so $f_{G W}$ is twice the spin frequency 17 . For every LMXB in Fig. 1 except the weakest one, the estimated rotation frequency is based on QPO splittings rather than NCOs, which means that about half of the frequencies might be double these estimates: $\sim 1200 \mathrm{~Hz}$ for density inhomogeneities and $\sim 800 \mathrm{~Hz}$ for $\mathrm{r}$-mode sloshing, rather than $\sim 600 \mathrm{~Hz}$. Doubling the frequency above $600 \mathrm{~Hz}$ reduces the emission amplitude by a factor 2 (at fixed X-ray flux assuming a steady-state torque balance), and increases the amplitude noise $S_{h}^{1 / 2}$ in an advanced NB IFO by a factor $\simeq \sqrt{2}$. As a result, at $\sim 1200 \mathrm{~Hz}$ frequency, Sco X-1 would still be readily detectable in a NB IFO but not in a WB IFO, and the strongest of the other LMXB's would be marginal. By contrast, if the frequencies are $\sim 600 \mathrm{~Hz}$ or $\sim 400$ $\mathrm{Hz}$ and the waves are in a steady state, then Sco X-1 should be very easily detected by a WB IFO, and several LMXB's should be detectable by narrow banding (NB).

The advanced IFOs can perform interesting searches for waves from known radio pulsars, such as the Crab and Vela for which the current upper limits (based on the pulsars' observed spindown rates) are shown as triangles. A Crab search, using coherent integrations based on the star's observed (slightly wandering) rotation period, could improve the limit on the Crab's wave amplitude by a factor 100 and constrain the star's gravitational ellipticity to $\epsilon \lesssim 7 \times 10^{-6}$ - which is approaching the realm of physically plausible ellipticities, $\epsilon \lesssim 10^{-6}$. (We shall discuss the physical origins of neutron-star ellipticities and their plausible ranges in Sec. 2.7.)

More interesting will be searches for waves from known, fast pulsars, since the signal strength scales as $\tilde{h}_{s} \propto \epsilon f^{2} / r$ (where $r$ is distance to the source). The up-sloping short-dashed lines in Fig. 1 show some examples of signal strengths. With a narrow-band IFO tuned to the vicinity of such a fast pulsar, the waves would be detectable when $\epsilon \gtrsim 2 \times 10^{-6}(100 \mathrm{~Hz} / f)^{2}(r / 10 \mathrm{kpc})$, which is in the realm of plausible ellipticities for pulsars throughout our galaxy so long $f$ exceeds $200 \mathrm{~Hz}$, i.e. the spin frequency exceeds $100 \mathrm{~Hz}$.

Also of great interest will be searches for previously unknown spinning neutron stars, for which the signal strengths $\tilde{h}_{s}$ will be reduced by a factor of a few to $\sim 15$ by the lack of prior information about the frequency and its evolution and the direction to the source (which determines the time-evolving doppler shift produced by the earth's motion) 222. 20 . A tunable, narrow-band IFO will be crucial to such searches. One can search more

cutlerThorne: submitted to World Scientific on February 5, 2008 
deeply by a factor of several, using a narrow-banded IFO that dwells on a given frequency and its neighborhood for a few days or weeks and then moves on to another frequency, than using a broad-interferometer that collects signal at all frequencies simultaneously for a year. Such searches will be in the band of physically plausible ellipticities, for stars throughout our galaxy, if $f \gtrsim 400$ $\mathrm{Hz}$ (spin frequency above $\sim 200$ revolutions per second).

One can search for a stochastic background of gravitational waves by cross correlating the outputs of LIGO's two $4 \mathrm{~km}$ IFOs 23 . For such a search the signal strengths $\tilde{h}_{s}(f)$ are shown in Fig. 1 as downward-sloping dotted lines, assuming a cross-correlation of 4 months of (not necessarily contiguous) data, and isotropic waves. The lines are labeled by the waves' energy density $\Omega(f)$ in a bandwidth equal to frequency and in units of the density to close the universe, $\Omega(f)=\left(f d E_{\mathrm{GW}} / d f\right) / \rho_{\text {closure }}$. Unfortunately, the frequency of optimal a priori sensitivity, $f \sim 70 \mathrm{~Hz}$ (where the noise curve is parallel to the dotted $\Omega$ lines), is near the center of a dead band for LIGO. This dead band arises from the fact that $1 / 70 \mathrm{~Hz} \simeq 14 \mathrm{~ms}$ is about the round-trip gravity-wave travel time between the two LIGO sites 24 . The result is a net debilitation of the stochastic background sensitivity by a factor of a few: the initial IFOs can detect an isotropic background with $\Omega$ down to $\sim 10^{-5}$, while advanced, wide-band IFOs can reach down to $\Omega \sim 5 \times 10^{-9}$, and a factor $\sim 2$ lower than this if it is reoptimized for low frequencies. These are interesting sensitivities, able to test a wide range of speculations about the physics of the early universe and perhaps even detect waves - but not able to reach the level predicted by standard inflation, $\Omega \lesssim 10^{-15}$. Such a detection could have profound implications for physics and cosmology; see Sec. 2.9.

Other waves that advanced IFOs in LIGO will seek and may detect are those from the stellar core collapse that triggers supernovae and the boiling of the nascent neutron star at the endpoint of that collapse (Sec. 2.6), accretion induced collapse of white dwarfs (Sec. 2.6), and totally unknown sources.

We now give a more detailed discussion of each of the high-frequency sources, focusing especially on event rate estimates and the information that the waves may bring. Box 1 gives a brief summary of the various sources and their detectability by LIGO I and II (WB and NB).

\subsection{Inspiraling $N S$ and $B H$ Binaries with $M_{B H} \lesssim 10 M_{\odot}$}

As we have discussed, wide-band advanced-LIGO (LIGO-II) IFOs can detect the waves from NS/NS inspirals out to $300 \mathrm{Mpc}$, NS/BH out to $650 \mathrm{Mpc}$, and $\mathrm{BH} / \mathrm{BH}$ out to $z=0.4$. The event rates out to these distances can be esti-

cutlerThorne: submitted to World Scientific on February 5, 2008 


\section{Box 1}

Brief Summary of Detection Capabilities of Advanced LIGO (LIGO-II) Interferometers

- Inspiral of NS/NS, NS/BH and BH/BH Binaries: The table below 25,15 shows estimated rates $\mathcal{R}_{\text {gal }}$ in our galaxy (with masses $\sim 1.4 M_{\odot}$ for $\mathrm{NS}$ and $\sim 10 M_{\odot}$ for $\mathrm{BH}$ ), the distances $\mathcal{D}_{\mathrm{I}}$ and $\mathcal{D}_{\mathrm{WB}}$ to which initial IFOs and advanced WB IFOs can detect them, and corresponding estimates of detection rates $\mathcal{R}_{\mathrm{I}}$ and $\mathcal{R}_{\mathrm{WB}}$; Sec. 2.3.

\begin{tabular}{lllll}
\hline \hline & NS/NS & NS/BH & BH/BH in field & BH/BH in clusters \\
$\mathcal{R}_{\text {gal }}, \mathrm{yr}^{-1}$ & $10^{-6}-5 \times 10^{-4}$ & $\lesssim 10^{-7}-10^{-4}$ & $\lesssim 10^{-7}-10^{-5}$ & $\sim 10^{-6}-10^{-5}$ \\
$D_{\mathrm{I}}$ & $20 \mathrm{Mpc}$ & $43 \mathrm{Mpc}$ & 100 & 100 \\
$\mathcal{R}_{\mathrm{I}}, \mathrm{yr}^{-1}$ & $3 \times 10^{-4}-0.3$ & $\lesssim 4 \times 10^{-4}-0.6$ & $\lesssim 4 \times 10^{-3}-0.6$ & $\sim 0.04-0.6$ \\
$D_{\mathrm{WB}}$ & $300 \mathrm{Mpc}$ & $650 \mathrm{Mpc}$ & $z=0.4$ & $z=0.4$ \\
$\mathcal{R}_{\mathrm{WB}}, \mathrm{yr}^{-1}$ & $1-800$ & $\lesssim 1-1500$ & $\lesssim 30-4000$ & $\sim 300-4000$ \\
\hline \hline
\end{tabular}

- Tidal disruption of NS by $\mathbf{B H}$ in NS/BH binaries: First crude estimates suggest WB IFOs can measure onset of disruption at $140 \mathrm{Mpc}$ well enough to deduce the NS radius to $15 \%$ accuracy (compared to current uncertainties of a factor $\sim 2$ ); see table above for rates; Sec. 2.4.

- BH/BH merger and ringdown: Rough estimates suggest detectability, by WB IFOs out to the cosmological distances shown in Fig. 2(b); rates for $\mathrm{BH} / \mathrm{BH}$ total mass $\sim 20 M_{\odot}$ are in table above; rates for much larger masses are unknown; Sec. 2.5.

- Low-Mass X-Ray Binaries: If accretion's spin-up torque on NS due is counterbalanced by gravitational-wave-emission torque, then WB IFOs can detect Sco X-1, and NB IFOs can detect $\sim 6$ other known LMXB's; Sec. 2.7.

- Fast, Known Spinning NS's (Pulsars with pulse frequency above $100 \mathrm{~Hz}$ ): Detectable by a advanced NB IFO in 3 months' integration time, if NS ellipticity is $\epsilon \gtrsim 2 \times 10^{-8}(1000 \mathrm{~Hz} / f)^{2}(r / 10 \mathrm{kpc})$, where $f$ is gravity wave frequency (twice the pulsar frequency) and $r$ is distance; actual ellipticities are unknown, but plausible range is $\epsilon \lesssim 10^{-6}$; Sec. 2.7.

- Fast, Unknown Spinning NS's: Unknown frequency wandering and doppler shifts degrade the detectable ellipticity $\epsilon$ by a factor of a few to $\sim 15$, so detection with a NB IFO requires $\epsilon \gtrsim(0.6$ to $3) \times 10^{-5}(100 \mathrm{~Hz} / f)^{2}(r / 10 \mathrm{kpc})$; Secs. 2.2 and 2.7 .

- Centrifugally Hung-Up Proto Neutron Stars in White-Dwarf Accretion-Induced Collapse and in Supernovae: Dynamics of star very poorly understood; if instability deforms star into tumbling bar, may be detectable by WB IFOs to $\sim 20 \mathrm{Mpc}$ (the Virgo Cluster), and possibly farther; event rates uncertain but could be enough for detection; Sec. 2.6.

- Convection of Supernova Core: May be detectable by WB IFOs, via correlations with neutrinos, for supernovae in our Galaxy and possibly Magellanic Clouds; Sec. 2.6.

- Gamma Ray Bursts: If triggered by NS/BH mergers, a few per year could be detectable by WB IFOs; if none are seen individually, statistical studies could nevertheless confirm gravity-wave emission by the gamma-burst triggers; Sec. 2.8.

- Stochastic Background: Detectable by cross correlating Hanford and Livingston $4 \mathrm{~km}$ detector outputs, if $\Omega=$ (gravitational-wave energy in $\Delta f \sim f \sim 40 \mathrm{~Hz}$ ) $z 5 \times 10^{-9}$; there are many possible sources of such waves in very early universe, all very speculative; Sec. 2.9. 
mated from observational data in our own galaxy 25, and an extrapolation out through the universe based on the density of massive stars (which can be deduced by several different metheds) 25.26.27. The resulting rates (as compiled and evaluated by V. Kalogera 25 ) are quite uncertain but very promising; see Box 1.

For NS/NS binaries, the event rate in our galaxy is constrained by the results of radio astronomers' searches for binary pulsars that will merge, due to gravitational radiation emission, in less than the age of the universe, and by other aspects of pulsar searches 25 . The resulting constraints, $10^{-6} / \mathrm{yr}$ $\lesssim \mathcal{R}_{\text {gal }} \lesssim 5 \times 10^{-4} / \mathrm{yr}$, extrapolate to a NS/NS event rate for advanced IFOs between $1 / \mathrm{yr}$ and $800 / \mathrm{yr}$. Searches in our galaxy for NS/BH binaries in which the NS is a pulsar have failed to find any as yet, so we must turn to much less reliable estimates based on "population synthesis" (simulations of the evolution of a population of progenitor binary systems to determine the number that make NS/BH binaries compact enough to merge in less than the universe's age). Population synthesis gives a NS/BH event rate in our galaxy in the range $\sim 10^{-7} / \mathrm{yr} \lesssim \mathcal{R}_{\text {gal }} \lesssim 10^{-4} / \mathrm{yr} 28$, though it is possible the rate could be even less than this (hence the $\lesssim 10^{-7} / \mathrm{yr}$ in Box 1). Extrapolating out into the universe, Kalogera finds a NS/BH rate in WB advanced IFOs between $\lesssim 1 / \mathrm{yr}$ and about 1500/yr. A similar analysis for BH/BH binaries based on population synthesis (third column of the table in Box 1) gives a rate between $\lesssim 30 /$ yr and $\sim 4000 /$ yr 29 . That population synthesis estimate ignores the likely role of globular clusters and other types of dense star clusters as "machines" for making BH/BH binaries 30: single black holes, being heavier than most stars in a globular, sink to the center via tidal friction, find each other, and make binaries; then the $\mathrm{BH} / \mathrm{BH}$ binaries get "hardened" (made more compact) by interaction with other black holes, reaching sizes where gravitational-wave emission will cause them to merge in less than the age of the universe; and further interactions will often eject the $\mathrm{BH} / \mathrm{BH}$ binaries from the globular, to interstellar space where they merge. Simulations by McMillan and Portegies Zwart 30 suggest that each dense cluster will make a number of such $\mathrm{BH} / \mathrm{BH}$ binaries, and extrapolations into the universe predict an event rate in WB advanced IFOs between $\sim 300$ and $\sim 4000 / \mathrm{yr}$ - though the uncertainties are probably larger than suggested by these numbers from the literature 3025.

These event rates are very encouraging. They make it seem quite likely that the advanced IFOs will observe tens to thousands of $\mathrm{BH}$ and NS inspirals per year, while the initial IFOs will be lucky to observe $\sim 1$ per year.

The observed inspiral waves will last for between $\sim 1000$ and 10, 000 cycles depending on the binary's masses, and will carry detailed information about

cutlerThorne: submitted to World Scientific on February 5, 2008 
the binary and about general relativistic deviations from Newtonian gravity. This information can be extracted with good precision using the method of matched filters. Specifically (denoting by $M=M_{1}+M_{2}$ the binary's total mass and $\mu=M_{1} M_{2} / M$ its reduced mass): 3132

(i) The binary's chirp mass $M_{c} \equiv \mu^{3 / 5} M^{2 / 5}$ will typically be measured, from the Newtonian part of the signal's upward frequency sweep, to $\sim 0.04 \%$ for a NS/NS binary and $\sim 0.3 \%$ for a system containing at least one $\mathrm{BH}$. (ii) If we are confident (e.g., on a statistical basis from measurements of many previous binaries) that the binary's spins are a few percent or less of the maximum physically allowed, then the reduced mass $\mu$ will be measured to $\sim 0.5 \%$ for NS/NS and NS/BH binaries, and $\sim 2 \%$ for $\mathrm{BH} / \mathrm{BH}$ binaries. (iii) Because the frequency dependences of the (relativistic) $\mu$ effects and spin effects are not sufficiently different to give a clean separation between $\mu$ and the spins, if we have no prior knowledge of the spins, then the $\operatorname{spin} / \mu$ correlation will worsen the typical accuracy of $\mu$ by a large factor, to $\sim 50 \%$ for NS/NS, $\sim 90 \%$ for $\mathrm{NS} / \mathrm{BH}$, and a factor $\sim 1.5$ for $\mathrm{BH} / \mathrm{BH}$. These worsened accuracies should be improved significantly (though we do not yet know how much) by waveform modulations due to spin-induced precession of the orbit $33,34,35$, and even without modulational information, a certain combination of $\mu$ and the spins will be determined to a few per cent. (iv) The distance to the binary ("luminosity distance" at cosmological distances) can be inferred, from the observed waveforms, to a precision $\sim 3 / \rho \lesssim 30 \%$, where $\rho \equiv S / N$ is the amplitude signal-to-noise ratio in the total LIGO network (which must exceed about 8 in order that the false alarm rate be less than the threshold for detection). (v) With the aid of VIRGO, the location of the binary on the sky can be inferred, by time of flight between the detector sites, to a precision of order five degrees 36 . (This angular resolution is rather worse than one might expect from a simple estimate based on time-of-flight alone. The reason is that because two LIGO detectors are nearly parallel, the addition of VIRGO to the network still leaves a near-degeneracy between polarization and time-of-flight information. The addition of a fourth detector with comparable sensitivity in Australia would decrease the error box on the sky $\Delta \Omega$ by a factor $\sim 4$. 6 .)

Advanced LIGO will likely produce a catalog of hundreds or thousands of binary inspirals and their inferred parameters; this catalog will be a valuable data base for observational astronomy and cosmology.

Important examples of the general relativistic effects that can be detected and measured with precision, in the inspiral waves, are these: (i) As the waves emerge from the binary, some of them get backscattered one or more times off the binary's spacetime curvature, producing wave tails. These tails act back on the binary, modifying its radiation reaction force and thence its inspiral rate 
in a measurable way. 37 (ii) If the orbital plane is inclined to one or both of the binary's spins, then the spins drag inertial frames in the binary's vicinity (the "Lense-Thirring effect"), this frame dragging causes the orbit to precess, and the precession modulates the waveforms 33 . This precession and modulation should be very strong in a significant fraction of NS/BH binaries 28 .

\subsection{Tidal Disruption of a NS by a BH: Measuring the Nuclear Equation of State}

As a NS/BH binary spirals inward, its NS experiences ever increasing tidal forces from the BH's gravitational field (its spacetime curvature). In many cases these tidal forces may tear the NS apart before it begins its final, quick plunge into the hole's horizon. The gravitational waves from this tidal disruption and from the termination of inspiral should carry detailed information about the NS's equation of state (the equation of state of bulk nuclear matter at $\sim 10$ times the density of an atomic nucleus). The disruption waves lie largely in the frequency band $\sim 300 \mathrm{~Hz} \lesssim f \lesssim 1000 \mathrm{~Hz}$, where the wide-band, advanced IFOs have good sensitivity, and where narrow-band or some other optimized IFO can do even better. This suggests that the advanced IFOs may be able to extract new information about the nuclear equation of state from the tidal-disruption waves. A first, crude estimate 38 suggests, for example, that for NS/BH binaries at $140 \mathrm{Mpc}$ distance (where the event rate could be a few per year; see the table in Box 1), tidal-disruption observations may enable the NS radius $R$ to be measured to a precision $\sim 15 \%$, by contrast with its present uncertainty (for fixed NS mass) of about a factor 2. From the measured radii would follow the desired equation-of-state information. Detailed numerical-relativity simulations will be required to firm up this estimate, and will be essential as a foundation for interpreting any tidal-disruption waves that are observed.

The merger waves from NS/NS binaries, by contrast with NS/BH, are likely to lie outside the band of good advanced-IFO sensitivity - at frequencies $f \gtrsim 1500 \mathrm{~Hz}$. However the onset of NS/NS merger, triggered by a plunge of the two NS's toward each other, may produce a strong "cliff" in the waves' spectrum, in a range $f \sim 400-1000 \mathrm{~Hz}$ of good sensitivity, and by measuring the cliff frequency we may strongly constrain the NS radii (assuming the NS masses have been determined accurately from the earlier portion of the waveform), and hence also learn about the nuclear equation of state 39 .

cutlerThorne: submitted to World Scientific on February 5, 2008 


\subsection{BH/BH Mergers and Ringdown: Observing the Nonlinear Dynamics of Spacetime Curvature}

A BH/BH binary evolves through three epochs: a gradual, adiabatic inspiral driven by gravitational radiation reaction (discussed in Sec. 2.3 above); a merger epoch, during which the holes' spacetime geometry undergoes violent, highly nonlinear oscillations; and a ringdown epoch, during which the merged black hole undergoes damped pulsations as it settles down into its final, Kerrmetric, quiescent state. The inspiral waves are rather well understood (Sec. 2.3), as are the ringdown waves; but the merger dynamics and waves are hardly understood at all.

It is likely that in many cases the holes are spinning rapidly and the system's three angular momentum vectors (two spins and one orbital) are substantially misaligned. In such cases, the merger dynamics might be quite rich, with spin-spin and spin-orbit coupling perhaps strong enough to produce spin flips and complex contortions and flows of the spacetime geometry as the two holes convert themselvesinto one.

Flanagan and Hughes 40.11 have made plausible estimates about the unknown aspects of the waves from each epoch - inspiral, merger and ringdown - and have used these estimates to evaluate the signal-to-noise ratios in LIGO and LISA for equal-mass, fast-spinning BH binaries. Figures 2 and 3 show their results for the initial (LIGO-I) and advanced (LIGO-II) IFOs, and Fig. 司(Sec. 3.3 below) shows them for LISA. [The LIGO-II results in Fig. 3 are updates 5 of the original Flanagan-Hughes results, taking account of the currently planned noise curve for LIGO-II, Fig. 1.]

Figures 2 and 3 suggest that, for $M \lesssim 24 M_{\odot}$ (two $12 M_{\odot}$ holes), the inspiral waves produce most of the signal in LIGO; for $24 M_{\odot} \lesssim M \lesssim 200 M_{\odot}$ the merger waves dominate; and for $200 M_{\odot} \lesssim M \lesssim 1000 M_{\odot}$, the merger and ringdown waves have roughly equal strength. (We say "suggest" because the plausible assumptions made by Flanagan and Hughes are far from secure; numerical-relativity simulations and/or observations are needed to firm them up.) For $M=20 M_{\odot}$ (two $10 M_{\odot}$ holes), LIGO's initial and advanced IFOs can detect the waves out to $100 \mathrm{Mpc}$ and $z=0.4$ respectively, at which distance population synthesis calculations suggest event rates $\lesssim 1$ /year and $\sim 30$ to $\sim$ 4000/year respectively (Box 1 and Sec. 2.3 above). Black-hole binaries more massive than $M \sim 50 M_{\odot}$ (two $25 M_{\odot}$ holes) are unlikely to form from main-sequence progenitors, but could well form in dense star clusters. The event rates at the distances seen by the initial IFOs $(\sim 200$ to $800 \mathrm{Mpc}$ for masses up to $\sim 1000 M_{\odot}$ ) and by the advanced IFOs (redshifts $z \sim 1$ to 2 for masses up to $\sim 2000 M_{\odot}$ ) are exceedingly uncertain; but for the advanced

cutlerThorne: submitted to World Scientific on February 5, 2008 


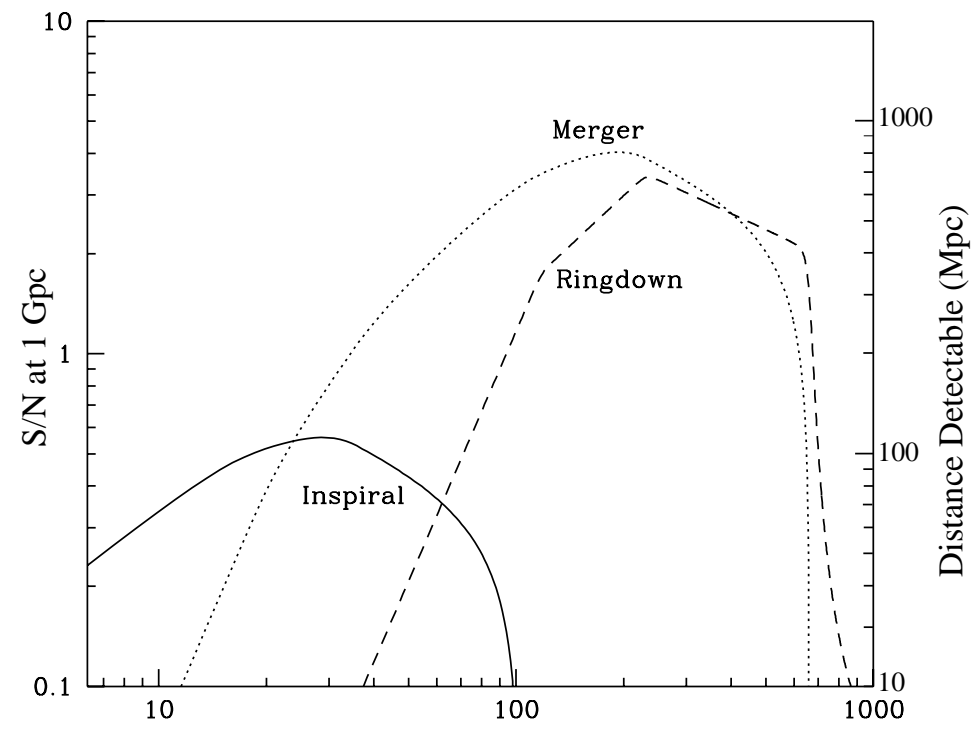

Figure 2. The inspiral, merger, and ringdown waves from equal-mass black-hole binaries as observed by LIGO's initial interferometers: The distance to which the waves are detectable (right axis) and the signal-to-noise ratio for a binary at $1 \mathrm{Gpc}$ (left axis), as functions of the binary's total mass (bottom axis). (Figure adapted from Flanagan and Hughes 4 .)
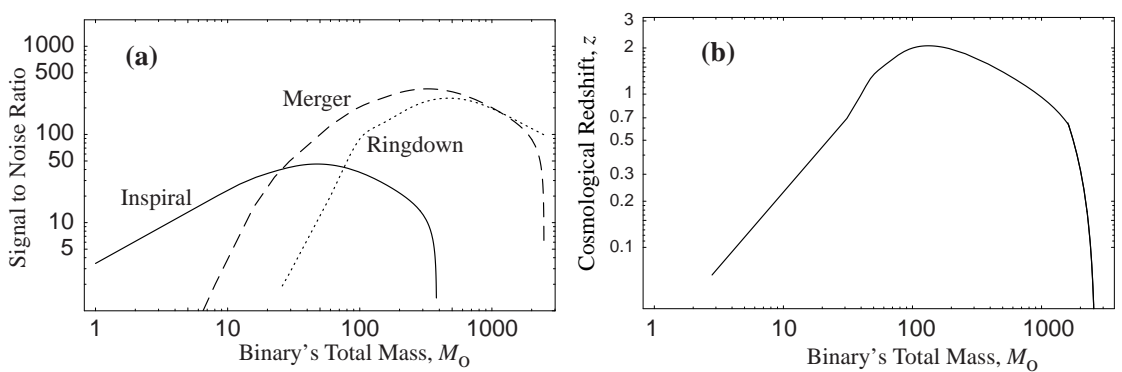

Figure 3. (a) The signal-to-noise ratio for the inspiral, merger and ringdown waves from fast-spinning, equal-mass black-hole binaries at a fiducial distance of $1 \mathrm{Gpc}$, as observed by advanced (LIGO-II) interferometers. (b) The cosmological redshift out to which these waves can be seen, assuming a cosmology with Hubble expansion rate $H_{O}=65 \mathrm{~km} / \mathrm{s} / \mathrm{Mpc}$, cold-matter density 0.4 of that required to close the universe $\Omega_{M}=0.4$, and racuum energy density (cosmological constant) 0.6 of closure, $\Omega_{\Lambda}=0.6$. (Figure from Ref. LIGO-II wide-band noise curve in Fig. 1 and the analysis of Flanagan and Hughes 10 .) 
IFOs they could be large.

\subsection{Supernovae and Accretion-Induced Collapse of White Dwarfs}

Type-II supernovae are triggered by the violent collapse of a stellar core to form a NS or BH. Despite decades of extensive modeling, we still understand only poorly the details of the collapse and how it triggers the explosion, and the birth throes of the NS or BH. The optical display brings little information, as the stellar material is so optically thick that the display is produced hours after the collapse and from radii $\sim 10,000$ times larger than the NS or BH. Neutrinos bring information about the core's temperature during the first few seconds after the collapse, and gravitational waves could reveal the nonspherical dynamics of the core's mass distribution on millisecond timescales and longer, and the nature of the core's final state: BH or NS.

Numerical models of supernovae suggest that, even if it is slowly rotating, a newborn proto-neutron-star will be convectively unstable, and that the gravitational waves from the convective overturn in the first $~$ one second of the star's life may be detectable throughout our galaxy and its orbiting companions, the Magellanic Clouds 42 . Although the supernova rate is low in our galaxy and its companions ( $\lesssim 1 / 30 y \mathrm{yrs})$, one observed event could be very valuable scientifically: The bulk of the supernova's neutrinos are thought to come from the same convecting material as produces the gravitational waves, so there should be correlations between the neutrinos and the waves, which could teach us much about the proto-neutron-star's dynamics.

Observations show that many type-II supernovae produce neutron stars and give them kicks of magnitude as large as $\sim 1000 \mathrm{~km} / \mathrm{s}$. Indeed about half of all radio pulsars are born with a kick larger than $500 \mathrm{~km} / \mathrm{s} 43$. The observed kicks suggest that at least some newborn proto-neutron-stars may be strongly asymmetric, perhaps due to fast rotation, and therefore could produce significant gravitational radiation. Rapidly rotating proto-neutronstars may also be produced by the accretion-induced collapse of white dwarf stars (AIC) in some cases, depending on the white-dwarf composition, central density and accretion rate 44 .

If the newborn proto-neutron-star in an AIC or supernova is spinning so fast that it hangs up centrifugally at a radius large compared to that of the final NS, then it may be dynamically (or at least secularly) unstable to deforming into a bar-shaped object that tumbles end-prer-end, emitting gravitational waves in LIGO's band of good sensitivity 4 . Recent simulations suggest that the bar will be long-lived, rather than just wrapping itself up into an axisymmetric shape and disappearing $\Theta$. 47 . The simulations show

cutlerThorne: submitted to World Scientific on February 5, 2008 
that the waves from such a bar may sweep upward in frequency, due to a gradual shrinkage of the proto-neutron-star, or may sweep downward due to development of "Dedekind-like" internal circulation 48 . The discovery of the waves from such a proto-neutron-star and observations of their frequency evolutions (which should mirror the bar's evolution) would teach us much. Though the strengths of the waves and the best signal processing techniques and thresholds are all ill-understood, a rough estimate 49 suggests that the advanced IFOs' range for detection might be the distance of the VIRGO cluster of galaxies (about $15 \mathrm{Mpc}$ ) and conceivably significantly larger, suggesting event rates that could be some per year but might be far less.

If the advanced WB IFOs detect no gravitational waves from a supernova at distance $r$, during a time $T$ preceeding the beginning of the optical outburst or over a time $T$ during a neutrino outburst, then one can thereby place a limit on the emitted gravitational-wave energy. The limit (as devised by Finn50) is based on the statistics of a cross correlation of the outputs of the two $4 \mathrm{~km}$ LIGO interferometers, over the chosen time $T$ and over some frequency band near the minimum of the advanced LIGO noise curve. In the band from $\sim 100$ to $300 \mathrm{~Hz}$ this limit is $\Delta E_{\mathrm{GW}} \lesssim 0.05(r / 15 \mathrm{Mpc})^{2}(T / 1 \mathrm{~h})^{1 / 2} M_{\odot}$. In the case of a neutrino-emitting supernova in our own galaxy, with $T \sim 1 \mathrm{sec}$, this limit is very impressive: $\sim 10^{-9} M_{\odot}$.

\subsection{Spinning Neutron Stars}

As a newborn neutron star settles down into its final state, its crust solidifies (crystalizes). The solid crust will assume nearly the oblate axisymmetric shape that centrifugal forces are trying to maintain. Thereafter the crust has a preferred shape (oblate about some preferred axis). One can define the NS's deformation ellipticity $\epsilon_{d}$ as the residual ellipticity the NS would then maintain (due to induced crustal shear stresses) if the NS were spyndown to zero frequency without the crust breaking or otherwise relaxing $515_{22}$ :

$$
\epsilon_{d} \sim 7 \times 10^{-8}\left(\frac{\nu_{\mathrm{rel}}}{\mathrm{kHz}}\right)^{2}
$$

where $\nu_{\text {rel }}$ is the spin frequency for which the crust is (most) relaxed. Again, this is the level of ellipticity that is "frozen into" the crust, as opposed to the ellipticity that arises from centrifugal forces and so "follows" the star's spin . If the star's angular momentum $\vec{J}$ deviates from the crust's preferred symmetry axis, the NS will wobble as it spins, with small "wobble angle" $\theta_{w}$. The NS will emit GW's with $h \propto \theta_{w} \epsilon_{d}$ at frequency $f_{G W}=f_{\text {rot }}+f_{\text {prec }}$, where the body frame precession frequency $f_{\text {prec }}\left[=\epsilon_{d} f_{\text {rot }}\left(I_{\text {crust }} / I\right)\right]$ is orders $-f$ magnitude smaller than $f_{\text {rot }}$; thus $f_{G W}$ is just slightly higher than $f_{\text {rot }}$ 53.51. The NS

cutlerThorne: submitted to World Scientific on February 5, 2008 
may also deviate slightly from axisymmetry about its principal axis; i.e., it may have a slight ellipticity $\epsilon_{e} \equiv\left(I_{1}-I_{2}\right) / I_{3}$ in its equatorial plane. It will then emit GW's with $h \propto \epsilon_{e}$ at twice the rotation frequency, $f_{G W}=2 f_{\text {rot }} 53$. The gravitational ellipticity $\epsilon$ referred to in Fig. 1 and in Sec. 2.2 is $\epsilon=\epsilon_{e}$ for deformations around the principal axis, and $\epsilon=2 \theta_{w} \epsilon_{d}$ for deformatins in which $\vec{J}$ is inclined to the preferred symmetry axis, producing wobble.

How large will be $\epsilon_{e}$ and $\theta_{w} \epsilon_{d}$ ? Ushomirsky et al. 18 have shown that, for typical NS parameters, $\epsilon_{e}<5 \times 10^{-7}\left(\sigma_{\max } / 10^{-2}\right)$, where $\sigma_{\max }$ is the (highly uncertain) crustal yield strain, estimated to be somewhere in the range $10^{-5}<\sigma_{\max }<10^{-1}$. Thus rough upper limits are $\epsilon_{e}<10^{-5}$ and [from Eq. 1] $\theta_{w} \epsilon_{d}<10^{-7}$. These upper limits are for ellipticities that are basically supported by crustal shear stresses, but there are other possible sources of ellipticity. For example, inside the superconducting cores of many neutron stars there may be trapped magnetic fields with mean strength $B_{\text {core }} \sim 10^{13} \mathrm{G}$ or even $10^{15} \mathrm{G}$. Because such a field is actually concentrated in flux tubes with $B=B_{\text {crit }} \sim 6 \times 10^{14} \mathrm{G}$ surrounded by field-free superconductor, its mean pressure is $p_{B}=B_{\text {core }} B_{\text {crit }} / 8 \pi$. This pressure could produce ellipticity $\epsilon_{\mathrm{e}} \sim \epsilon_{d} \sim p_{B} / p \sim 10^{-6} B_{\text {core }} / 10^{15} \mathrm{G}$ (where $p$ is the core's material pressure). We are extremely ignorant, and correspondingly there is much to be learned from searches for gravitational waves from spinning neutron stars.

From this discussion we see that the range of plausible gravitational ellipticities for a spinning neutron star is $\epsilon \lesssim 10^{-6}$, though they may get as large as $10^{-5}$. The strengths of the waves produced by such ellipticities are shown in Fig. 1 and the waves' detectability is discussed in Sec. 2.2.

Neutron stars gradually spin down, due in part to gravitational-wave emission but perhaps more strongly due to electromagnetic torques associated with their spinning magnetic fields and pulsar emission. This spin-down reduces the strength of centrifugal forces, and thereby causes the star's oblateness to decrease, with an accompanying breakage and resolidification of its crust's crystal structure (a "starquake") 54. In each starquake, $\theta_{w}, \epsilon_{e}$, and $\epsilon_{d}$ will all change suddenly, thereby changing the amplitudes and frequencies of the star's two gravitational "spectral lines" $f=2 f_{\text {rot }}$ and $f=f_{\text {rot }}+f_{\text {prec }}$. After each quake, there should be a healing period in which the star's fluid core and solid crust, now rotating at different speeds, gradually regain synchronism. By monitoring the amplitudes, frequencies, and phases of the two gravitational-wave spectral lines, and by comparing with timing of the electromagnetic pulsar emission, one might learn much about the physics of the neutron-star interior.

A spinning NS can radiate not only due to built-in asymmetries, as discussed above, but also due to a gravitational-radiation-reaction-driven insta-

cutlerThorne: submitted to World Scientific on February 5, 2008 
bility first discovered by Chandrasekhar 55 and elucidated in greater detail by Friedman and Schutz 56 . In this "CFS instability", density waves travel around the star in the opposite direction to its rotation, but are dragged forward by the rotation. These density waves produce gravitational waves that carry positive energy as seen by observers far from the star, but negative energy from the star's viewpoint; and because the star thinks it is losing negative energy, its density waves get amplified. This intriguing mechanism is similar to that by which spiral density waves are produced in galaxies Although the CFS instability was once thought ubiquitous for spinning stars 56.57 , we now know that neutron-star viscosity will kill it, stabilizing the star and turning off the waves, when the star's temperature is above some limit $\sim 10^{10} \mathrm{~K} 58$ and below some limit $\sim 10^{9} \mathrm{~K} 5$; and correspondingly, the instability should operate only during the first few years of a neutron star's life, when $10^{9} \mathrm{~K} \lesssim T \lesssim 10^{10} \mathrm{~K}$, and only for NS spins greater than $\sim 90 \%$ of the upper limit set by mass-shedding at the equator 60.

Shortly after GR15 there was a surge of renewed interest in the CFS instability when Andersson 61 and Friedman and Morsink 62 showed that r-modes (driven by Coriolis forces) are unstable atall spin values (again, in the absence of viscosity), and then Lindblom et al. 63 and Andersson et al. 64 estimated that in a newborn NS, the instability would not be damped by shear or bulk viscosity, but instead would rapidly spin down the NS to $\nu \lesssim 100 \mathrm{~Hz}$. However, since then, a host of other damping mechanisms have been suggested, including. (i) wind-up of magnetic fields 55 , (ii) dissipation at the crust-core bondary 66 , (iii) bulk viscous damping from weak interactions involving hyperons in the inner core, which is predicted to kill the instability unless the star is less masive than about $1.2 M_{\odot} 6766$; and (iv) nonlinear mode-mode coupling, specifically a 3 -mode interaction, which is predicted to damp out r-modes at a maximum dimensionless amplitude $\sim 10^{-3}$ ( spin frequency $\left./ 1000 \mathrm{~Hz}\right)^{5 / 2} 69$. It therefore appears that $\mathrm{r}$-modes will not be significant $\mathrm{GW}$ sources, after all.

\subsection{Gamma Ray Bursts}

Cosmic gamma-ray bursts, observed $\sim$ once per day by detectors on spacecraft, are thought to be produced by extremely hot shocks in a relativistic fireball, which is ignited by rapid inflow of gas onto a newborn black hole 0 . The fireball is so dense that electromagnetic observations cannot probe the BH's vicinity; the observed gamma rays are produced at radii $\sim 10^{13}$ to $10^{15}$ $\mathrm{cm}$ - millions to billions of times larger than the hole itself. The only hope for probing the fireball's trigger is via gravitational waves: The BH and its accretion flow are thought to form violently, by the collapse of a massive star (per-

cutlerThorne: submitted to World Scientific on February 5, 2008 
haps initiated by merger with a companion) ["a hypernova"], or by the merger of a binary made of compact objects: a NS/NS binary, a BH/NS binary, a $\mathrm{BH} /$ white-dwarf or a $\mathrm{BH} / \mathrm{He}$-core binary 6 . Each of these gamma-burst triggers should emit strong gravitational waves that carry detailed information about its source.

The distribution of $\gamma$-ray bursts is bimodal: there are long bursts (duration $\gtrsim 2 \mathrm{~s}$ ) and short bursts ( $\lesssim 2 \mathrm{~s}$ ). Beppo-Sax has detected afterglows from several long bursts, allowing their redshifts to be measured. No redshifts are known for short bursts, but their distance can be estimated from the $\left\langle V / V_{\max }\right\rangle$ statistic 71 (assuming a standard profile, and that their event rate tracks the star formation rate). The best event rate estimates (by M. Schmidt 71) currently are: $1 / \mathrm{yr}$ to $D=170\left(\theta_{0} / 0.1\right)^{2 / 3} \mathrm{Mpc}$ for long bursts, and $1 / \mathrm{yr}$ to $\mathrm{D}=250\left(\theta_{0} / 0.1\right)^{2 / 3} \mathrm{Mpc}$ for short bursts. Here the gamma-rays are assumed beamed into solid angle $2 \pi \theta_{0}^{2}$. Clearly, these rates (for $\theta_{0}=0.1$ ) are quite consistent with the estimated NS-NS merger rates given in Box 1, and marginally consistent with the BH-NS rate.

If BH-NS mergers are indeed responsible for either long or short bursts, the (WB) LIGO II detection rate would be $\sim 15-50 /$ yr. Of course, these closest sources will generallly not be seen in $\gamma$-rays, since they are typically beamed away from us; the closest burst for which we see the $\gamma$-rays (in 1 yr) is at $D=1.0(1.5) \mathrm{Gpc}$ for long (short) bursts. Could these be detected in coincidence with GW inspiral events? Box 1 states that LIGO II (WB) could detect BH-NS (NS-NS) inspirals to $0.65(0.30)$ Gpc. But those distances assume a random orientation of the source, and assume that $S / N>8$ is required for confident detection. If the $\gamma$-rays are beamed perpendicular to the orbital plane, then in cases where we detect the burst, we are also at optimal "viewing angle" for the GW's. The amplitude $h$ of the GW's beamed "up the axis" are $\sqrt{\frac{5}{2}} \sim 1.58$ larger than the rms value (averaged over the sky, as seen from the source). Also, the near-coincident detection of a $\gamma$-ray burst in time (within several seconds of each other) and on a consistent area of the sky, effectively reduces by another factor $\sim 1.5$ the $\mathrm{GW} \mathrm{S/N}$ required for detection with $99 \%$ confidence 2 . Thus the distances out to which LIGO I and II could detect binary mergers associated with $\gamma$-ray bursts are $\sim 2.4$ times farther than the distances given in Box 1; for LIGO II: $720 \mathrm{Mpc}$ for NS/NS and $1.55 \mathrm{Gpc}$ for NS/BH mergers. Thus, if NS/BH mergers are responsible for long and/or short bursts, with LIGO II one might expect $\sim 2 / \mathrm{yr}$ simultaneous detections in both GW's and $\gamma$-rays. If NS/NS mergers are responsible, one might expect to wait $\sim 5 \mathrm{yr}$.

M. van Putten $[3$ points out that in the above $\gamma$-ray burst scenarios, the ac-

cutlerThorne: submitted to World Scientific on February 5, 2008 
cretion torus itself might develop large non-axisymmetries and so be a strong source of GW's. He develops a picture of long bursts as due to "suspended" accretion around a rapidly rotating $\mathrm{BH}$, with most of the BH's angular momentum getting transferred to the torus via Maxwell stresses (similar to the Blandford-Znajek effect). If the torus develops "lumps", van Putten estimates that $\sim 10 \%$ of the BH's spin energy could get radiated away in GW's-so $E_{G W} \sim 0.3 M_{\odot}\left(M_{B H} / 10 M_{\odot}\right)$. He predicts a sinusoidal waveform with frequency $f \sim 1-2 \mathrm{kHz}$ that wanders slowly in time - which is simple enough to allow near-optimal data analysis. Would this be detectable? The rms $S / N$ would then be (taking $z \ll 1$ and approximating $f$ and $S_{h}(f)$ as constants over the signal band, for simplicity),

$$
\frac{S}{N}=\frac{\sqrt{2 E_{G W}}}{\pi D f^{1 / 2} h_{n}(f)} .
$$

yielding $S / N=1.4$ for $E_{G W}=0.3 M_{\odot}, D=150 \mathrm{Mpc}, f=10^{3} \mathrm{~Hz}$, and $h_{n}\left(\equiv \sqrt{5 f S_{h}(f)}\right)=8 \times 10^{-22}$ (LIGO II WB value at $1 \mathrm{kHz}$ ). This does not seem especially promising, but given the uncertainty in $E_{G W}$ and $D$ (for the closest source in one year), it seems worth looking for. Moreover, one could improve the sensitivity in LIGO-II by a factor of a few by reshaping the noise curve so it is lower at $f \sim 1 \mathrm{kHz}$, at the price of higher noise at lower frequencies (cf. the difference between the NB and WB LIGO-II curves in Fig. 1).

If gravitational waves are detected from one or more gamma-burst triggers, the waves will almost certainly reveal the physical nature of the trigger. Moreover, by comparing the arrival times of the gravitational waves and the earliest gamma rays, it should be possible to measure the relative propagation speeds of light and gravitational waves to an accuracy $\sim 1 \mathrm{sec} / 10^{10} \mathrm{yr} \sim 10^{-17}$.

If no gravitational waves are detected from any individual gamma burst, then (as Mohanty, Finn and Romano 74 have shown) the correlation between gamma bursts and gravitational waves might nevertheless be established by statistical studies of the advanced IFOs' gravitational-wave data in narrow time windows preceeding the gamma bursts.

\subsection{Sources of Stochastic Background}

Searches for a GW stochastic background with Earth-based detectors begin with the assumptions that 1) the GW background will certainly be smaller than the instrumental noise at all frequencies, and 2) the instrumental noise level will not be known sufficiently well $a$ priori that one can simply search

cutlerThorne: submitted to World Scientific on February 5, 2008 
for "excess noise" in each instrument. Thus, stochastic background searches with Earth-based detectors are based on cross-correlating the outputs of two highly independent detectors. (The two $4 \mathrm{~km}$ LIGO detectors at Hanford, Washington and Livingston, Louisiana give the best sensitivity).

The most plausible sources of a stochastic gravitational-wave background in LIGO's frequency band are processes in the very early universe. The current best limit on the strength of such waves is $\Omega \lesssim 6 \times 10^{-6}$, where $\Omega \equiv \int \Omega(f) d(\ln f)$ is the integrated GW energy density from all frequency bands. A wave energy larger than this would have caused the universe to expand too rapidly through the era of primordial nucleosynthesis (universe age $\sim$ a few minutes), thereby distorting the universal abundances of light elements away from their observed values. Other known bounds on $\Omega(f)$ are: 1) $h_{100}^{2} \Omega\left(f \sim 10^{-8} \mathrm{~Hz}\right)<10^{-8}$ from pulsar timing; and 2) $h_{100}^{2} \Omega(f)<7 \times 10^{-11}\left(f / H_{0}\right)^{-2}$ for $3 \times 10^{-18} h_{100} \mathrm{~Hz}<f<\times 10^{-16} h_{100} \mathrm{~Hz}$, from microwave background anisotropy measurements. Here $h_{100}$ is the Hubble constant in units of $100 \mathrm{~km} / \mathrm{s} / \mathrm{Mpc}$.

The LIGO II interferometers would improve the current limit at $f \sim 40$ $\mathrm{Hz}$ by a factor $\sim 10^{3}$, to $\Omega(f \sim 40 \mathrm{~Hz}) \simeq 5 \times 10^{-9}$ - an improvement enabling LIGO to test a number of current speculations about the very early universe. Inflationary models of the early universe predict that vacuum fluctuations, created in the Planck era when the universe was being born, should have been parametrically amplified, during the first $\sim 10^{-25}$ sec of the universe's life, to produce a stochastic gravitational wave background in the LIGO band. Unfortunately, if "standard" inflation theory is correct, then the amplified waves are much too weak for LIGO to detect, $\Omega \lesssim 10^{-15}$. 5 . The most plausible modifications of standard inflation push $\Omega$ downward from this 5 , but some less plausible modifications push it upward, to the point of detectability 76 .

The first, tentative efforts to combine superstring theory with inflationary ideas have produced a new description of the very early universe called the "pre-big-bang model", in which string effects cause the gravitational-wave spectrum to rise steeply at high frequencies - most likely at frequencies above LIGO's band, but quite possibly in or below that band 7 . The result could be waves strong enough for LIGO to detect. A non-detection would significantly constrain the pre-big-bang model.

There are a wide variety of postulated mechanisms that could have produced strong gravitational waves, with wavelengths of order the horizon size, at various epochs in the very early universe. Those waves (if any) produced at (universe age) $\sim 10^{-25} \mathrm{sec}$, corresponding to (universe temperature $T$ ) $\sim 10^{9}$ $\mathrm{GeV}$, would have been redshifted into the LIGO band today and might be detectable. The temperature (energy) region $\sim 10^{9} \mathrm{GeV}$ is tera incognita; 
LIGO's advanced detectors will provide our first opportunity for a serious experimental exploration of it. Among the speculated wave-generating mechanisms that could operate there, and that LIGO could constrain (or discover!), are these:

- A first-order phase transition in the states of quantum fields at $T \sim 10^{9}$ K. Such a phase transition would nucleate bubbles of the new phase that expand at near the speed of light and collide to produce gravitational waves; and their collisions would also generate turbulence that radiates waves. If the transition is strongly first order the waves would be strong enough for LIGO's advanced IFOs to detect 78 .

- Goldstone modes (coherent, classical excitations) of scalar fields that arise in supersymmetric and string theories. If strongly excited, these modes will entail coherent flows of energy that radiate gravitational waves strong enough for detection 99 .

- Coherent excitations of our $3+1$ dimensional universe, regarded as a "brane" (defect surface) in a higher dimensional universe. The excitations could be of a "radion" field that controls the size or curvature of the additional dimensions, or they could be of the location and shape of our universe's brane in the higher dimensions; in either case, if there is an equipartition of energy between these excitations, in the very early universe, and other forms of energy, then the excitations will produce gravitational waves easily strong enough for detection by LIGO's advanced IFOs. LIGO would thereby probe one or two additional dimensions of size or curvature length $\sim 10^{-10}-10^{-13} \mathrm{~mm}$; by contrast, LISA's lower-frequency observations would probe lengths $\sim 1-10^{-5} \mathrm{~mm}$. If the number of extradimensions is larger than 2 , the probes reach to much smaller scales 80.81

Cosmic strings (not to be confused with superstrings), produced in the early universe, were once regarded as candidates for seeding galaxy formation, but recent cosmological observations have ruled them out as seeds. Nevertheless, it remains possible that a network of vibrating cosmic strings too weak to seed galaxy formation was formed in the early universe. LIGO can search for the presence of such a network in two ways: (i) Via the stochastic background of gravitational waves that the strings' vibrations produce; this background would be strong enough for the advanced IFOs to detect if the strings' mass per unit length is $\gtrsim 10^{-8}$. 82 (ii) Via occasional, individually detectable, strong non-Gaussian bursts ("spikes") of gravitational waves produced by kinks or cusps along the string 33 .

cutlerThorne: submitted to World Scientific on February 5, 2008 
Cusps are points on the string that, at one instant, move at the speed of light; kinks typically arise when two strings cross each other and interconnect. We now briefly discuss the associated GW spikes as analyzed by Damour and Vilenkin 83. These signals have no free parameters, except the overall amplitude and the arrival time $t_{c}: h(t) \propto\left|t-t_{c}\right|^{1 / 3}$ for cusps and $h(t) \propto$ $\left|t-t_{c}\right|^{2 / 3}$ for kinks. The time spent in the detector band is $\sim 1 / f_{c}$ where $f_{c}$ is the center of the band. Matched filter searches should therefore be trivial, with coincidence between detectors (and other vetoes) used to remove instrumental bursts. Cusp bursts are more highly beamed than kink bursts, have higher amplitude, and are more detectable (the higher amplitude more than compensates for the decreased probability that any given cusp burst is beamed in our direction). Cusp bursts should be detectable for a large range of string tension $\mu$ not otherwise constrained: LIGO II should detect cusp bursts for $\mu \gtrsim 10^{-11}$, while LISA could detect $\mu \gtrsim 10^{-13}$. Burst searches thus provide a stronger limit on cosmic strings than searches for a stochastic, Gaussian background.

Each of these cosmological speculations is plausible, though not highly likely. (For further explication of most of them, we highly recommend the review by Allen 44.) Perhaps their greatest value is to remind us of how terribly ignorant we are of physics and astrophysics in the domain that LIGO's advanced IFOs will probe. Our ignorance may well be even greater than that of the pioneering radio astronomers of the 1930s and X-ray astronomers of the 1960s; and as there, so also here, the first waves to be discovered may well be from sources that were previously unknown. LIGO II and its partners could bring us a revolution of insights into the universe comparable to the revolutions wrought by radio and X-ray astronomy.

\section{Low-Frequency Sources Sought by LISA}

\subsection{LISA's Noise Curve and Conventions for Wave Strengths}

In discussing low-frequency sources, we shall place them in the context of the sensitivity of LISA, the Laser Interferometer Space Antenna 55 . As is discussed in Schutz's chapter in this volume 6 , LISA consists of three drag-free spacecraft at the corners of a 5-million-kilometer triangle. The spacecraft track the distances to each other using laser beams, thereby searching for gravitational waves in the $10^{-4}-1 \mathrm{~Hz}$ low-frequency band. LISA is tentatively scheduled for launch in 2011 as a joint European-American mission with a nominal lifetime of 5 years, though it could well continue to collect GW data much longer than that. By the time LISA begins its GW searches, the second-

cutlerThorne: submitted to World Scientific on February 5, 2008 


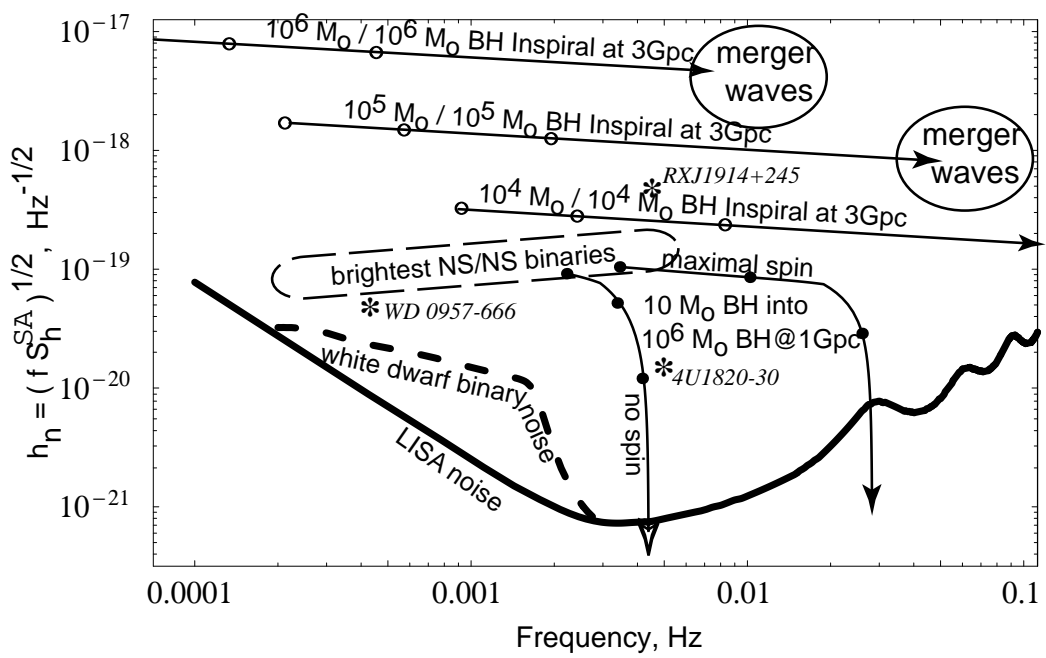

Figure 4. FISA's sky- and polarization-averaged rms noise $h_{n}(f)$ in a bandwidth equal to frequency, 6 compared with the strengths of the waves from several low-frequency sources. The wave strengths are plotted so the height above the noise curve is the $S / N$ for a wave search using optimal signal processing; see text for further detail. For frequency-sweeping waves (arrowed curves) the dots are, from left to right, the signal strength and frequency 1 year, 1 month, and one day before the end. The thick-dashed curve is a background of waves from WD-WD binaries that are so numerous they cannot be removed from the data, and so constitute a noise when searching for other waves.

generation earth-based detectors (e.g. the advanced LIGO-II IFOs) described in the last section could be nearing the end of their observations, and a transition to a third generation might be near at hand.

Figure 1 depicts LISA's noise and compares it with the wave strengths for various low-frequency sources, in much the same manner as Fig. 11 depicted LIGO's noise and compared it with high-frequency wave strengths. However, there are important differences in conventions between the LISA Fig. 1 and the LIGO Fig. 1:

First: For LIGO we plotted vertically the square root of the spectral density of the fractional arm-length difference, $\tilde{h}(f) \equiv \sqrt{S_{\Delta L / L}(f)}$ (noise per root $\mathrm{Hz}$ ) for a single $4 \mathrm{~km} \mathrm{IF0.} \mathrm{For} \mathrm{LISA,} \mathrm{by} \mathrm{contrast,} \mathrm{we} \mathrm{plot} \mathrm{the} \mathrm{rms}$ 
GW strain noise in a bandwidth equal to frequency $\Delta f=f$, averaged over the sky and over GW polarizations, for the full 3-spacecraft LISA system: $h_{n}=\sqrt{f S_{h}^{\mathrm{SA}}(f)}$, where $S_{h}^{\mathrm{SA}}(f)$ is the noise spectral density inverse-averaged over the sky and polarizations 8 . (For LIGO the analogous quantity is $h_{n}=$ $\sqrt{5 f} \tilde{h}$, where the $\sqrt{5}$ accounts for the average over the sky and polarizations.) In many discussions of LISA a different quantity is used to characterize the noise: the amplitude sensitivity to periodic sources for a one-year integration time and a signal-to-noise ratio of 5 , averaged over the sky and over source polarizations. That quantity, $h_{\mathrm{SN} 5,1 \mathrm{yr}}^{\mathrm{SA}}$, is related to our rms noise $h_{n}$ by

$$
h_{n}=\frac{\sqrt{f \times 1 \mathrm{yr}}}{5} h_{\mathrm{SN} 5,1 \mathrm{yr}}^{\mathrm{SA}} .
$$

Second: Our wave-strength convention for LISA (Fig. 1) is also different from that for LIGO (Fig. 1): The height of a source point or curve above the LISA noise curve is equal to the signal-to-noise ratio $S / N$ in a search for the source's waves using optimal signal processing. By contrast, for LIGO (Fig. 1), the height above the noise curve is the signal-to-threshold ratio $S / T$, with the threshold being that at which the false alarm probability is one per cent when using the best currently known, practical data analysis algorithm (typically $T \gtrsim 5 N$ ). The difference in convention is motivated by the fact that we know little as yet about practical LISA data analysis algorithms and thus don't know with much confidence how to set thresholds, whereas LIGO data analysis is fairly mature.

For LISA, when evaluating the $S / N$ 's that determine the height of the source points in Fig. 目, we assume for periodic sources (e.g. white dwarf binaries) an integration time of five years (the nominal mission lifetime), and for sources with a large frequency sweep, we assume at a given frequency $f$ an integration time that is the shorter of the time until the signal stops, and the time to sweep through a frequency band $\Delta f=f$.

Notice that LISA's minimum noise $h_{n} \simeq 10^{-21}$ is nearly the same as that of LIGO's first interferometers (Fig. 1 with $h_{n}=\sqrt{5 f} \tilde{h}$ ), but at 100,000 times lower frequency $f$. Since the waves' energy flux scales as $f^{2} h^{2}$, this corresponds to $10^{10}$ better energy sensitivity than LIGO-I.

LISA can detect and study, simultaneously, a wide variety of different sources scattered over all directions on the sky. The key to distinguishing the different sources is the different time evolution of their waveforms. The key to determining each source's direction, and confirming that it is real and not just noise, is the manner in which its waves' amplitude and frequency are modulated by LISA's complicated orbital motion - a motion in which the interferometer triangle rotates around its center and around the normal to the

cutlerThorne: submitted to World Scientific on February 5, 2008 
ecliptic plane once per year as the spacecraft orbit the sun; see the discussion by Schutz $\mathrm{g}$ elsewhere in this volume. Most sources will be observed for of order a year or longer, thereby making full use of these modulations.

Our understanding of sources and data analysis for LISA has evolved considerably since GR15. The principal sources are (i) short-period stellar-mass binaries - both galactic and extragalactic, (ii) the inspiral and merger of massive $\left(\sim 10^{6} M_{\odot}\right)$ BH binaries, (iii) the inspiral and capture of stellar-mass compact objects (white dwarfs, NS's and BH's) into massive BH's, (iv) the gravitational collape of supermassive stars, and (v) a stochastic GW background from the early universe. We discuss these in turn in the following sections.

\subsection{Short-Period Binaries}

Unlike the case for ground-based detectors, there are known GW sources that LISA is certain to detect at its planned sensitivity: short-period binary stars in our galaxy. There are currently about a dozen galactic binaries with GW frequencies above $0.1 \mathrm{mHz}$ that LISA would be able to detect and study in detail. These include: 8 (i) White-Dwarf / white-dwarf (WD/WD) binaries; the most detectable known example is WD 0957-666 (shown in Fig. A), with masses 0.37 and $0.32 M_{\odot}$, distance from Earth $100 \mathrm{pc}$, and time to merger $2 \times 10^{8}$ years. (ii) Am CVn stars - a white dwarf (WD) that accretes from a low-mass helium-star companion; the most detectable known example is RXJ1914+245 (shown in Fig. 1 ), a $0.07 M_{\odot}$ helium star orbiting a $0.6 M_{\odot}$ WD at 100pc distance from Earth. (iii) Low-mass X-ray binaries - a NS that accretes from a low-mass companion; the most detectable known example is

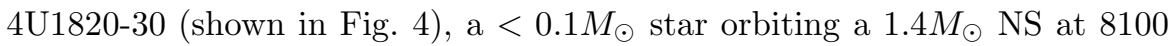
pc from Earth. These sources will provide an important test that LISA is functioning as expected (as well as a test of the general relativistic theory of GW emission, in the weak-gravity limit).

These optically-known binaries are just the tip of the iceberg; there are an estimated $10^{8-9}$ galactic binaries with GW frequencies $f>0.1 \mathrm{mHz}$ (most are $\mathrm{WD} / \mathrm{WD}$ ). At frequencies below $\sim 2 \mathrm{mHz}$, there are many binaries per resolvable frequency bin $\Delta f=1 / T_{\text {obs }} \sim 10^{-8} \mathrm{~Hz}$ - too many to be fit and removed from LISA's data, so they constitute a source of "confusion noise" that will dominate over the instrumental noise at these frequencies. Thus LISA truly suffers from an "embarrassment of riches." But there is also some science to be extracted from this confusion noise: because the WD/WD background is anisotropic, the confusion noise level is modulated by LISA's time-varying antenna pattern on the sky. From this one can extract with few-percent ac-

cutlerThorne: submitted to World Scientific on February 5, 2008 
curacy the $m=1$ and $m=2$ moments (in spherical coordinates centered on the Sun) of the WD/WD distribution 69.

Above $\sim 2 \mathrm{mHz}$ the density of sources thins to less than $\sim 0.5$ per frequency bin, and one expects to be able to measure individually all these binaries, about $10^{4}$ of them, and remove them from the data. The confusion noise therefore drops dramatically above $\sim 2 \mathrm{mHz}$ - to a level set by the extragalactic binary background (about 10 times smaller than the galactic background) - and crosses below the instrumental noise curve; see Fig. 4 .

Although it should be possible to remove the binaries above $2 \mathrm{mHz}$ from LISA's data so as to search there for other waves (e.g. from inspiral of small mass BH's into supermassive BH's; cf. Fig. (4), no effort has yet been put into the task of developing practical data analysis algorithms to perform the removal. Designing and optimizing such algorithms is an important challenge for the next few years.

For the $\sim 10^{3}$ highest-frequency binaries, one should be able to measure the binary's rate of change of frequency $\dot{f}$ (due to inspiral driven by GW emission). From the measured $\dot{f}$ and $f$ one can infer the binary's chirp mass $M_{c}$, and from $M_{c}$ and the measured amplitudes of the binary's two polarizations once can infer its inclination to the line of sight and its distance. For binaries with $f \sim 10^{-2} \mathrm{~Hz}$, one expects to measure the angular location to $\sim 1^{\circ} 9$ and the distance to $\sim 2 \%$ 11. Thereby one can obtain a 3-D map of the galaxy with distances unsullied by problems of dust, etc.

\subsection{Mergers of Supermassive Black Holes}

The merger of two supermassive black holes (SMBHs; $10^{4}-10^{7} M_{\odot}$ ) at $z=1$ could be detected by LISA with S/N of thousands; see Fig. 5 . This $\mathrm{S} / \mathrm{N}$ level is at least 10 times greater than we can expect with ground-based detectors (Figs. 2 and 3) - so high that the inspiral portion of the waveform could typically yield the binary parameters to the following accuracies: individual masses to $\Delta M_{i} / M_{i} \sim 10^{-4}$, the so-called spin-orbit parameter $\beta$ to $\Delta \beta \sim$ $10^{-3}$, the distance to $\Delta D / D \sim 10^{-3}-10^{-2}$, and the position of the source on the sky to $\sim 10^{-4} \mathrm{sr} 92$. Knowing the binary's initial conditions with such high accuracy, it should be possible to make high-precision comparisons of numerical relativity simulations with the observed merger waveforms thereby extending LIGO's exploration of the nonlinear dynamics of curved spacetime (Sec. 2.5) into a domain of far higher accuracy. For example, as the gravitational waves from a binary's merger depart from their source, the waves' energy should create (via the nonlinearity of Einstein's field equations) a secondary wave called the "Christodoulou memory" 93.94.95, which LISA

cutlerThorne: submitted to World Scientific on February 5, 2008 


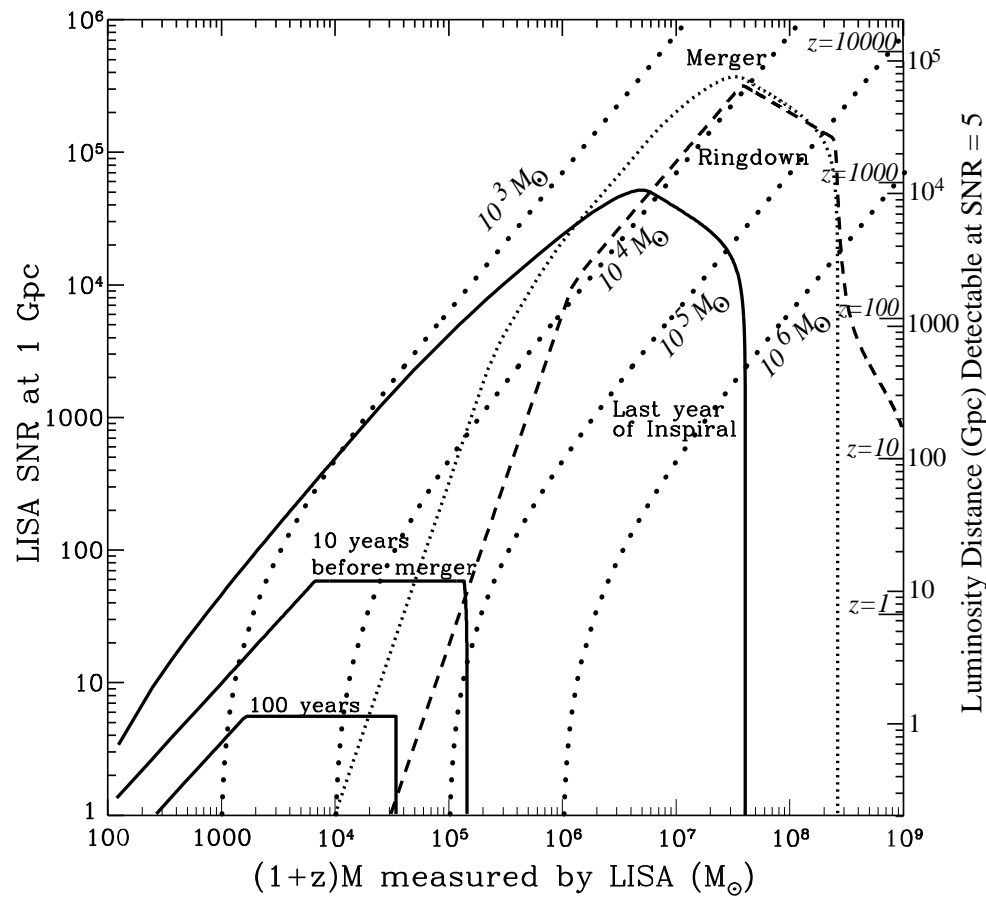

Figure 5. The waves from equal-mass, supermassive black-hole binaries as observed by LISA in one year of integration time. Plotted horizontally is the binary's total mass $M$ multiplied by 1 plus the binary's cosmological redshift $z$. The solid, dashed, and tight-dotted curves refer to the left axis, which shows the estimated $\mathrm{S} / \mathrm{N}$ for LISA's observations when the binary is $1 \mathrm{Gpc}$ from earth (redshift $z \simeq 0.2$ ). The wide-spaced dots are curves of constant binary mass $M$, for use with the right axis, which shows the luminosity distance and redshift to which the binary can be detected, with $\mathrm{S} / \mathrm{N}=5$ (look at the intersection of a wide-dotted curve with a solid, dashed, or tight-dotted curve and then project horizontally onto the right axis). (The assumed cosmology is $H_{O}=65 \mathrm{~km} / \mathrm{s} / \mathrm{Mpc}, \Omega_{\Lambda}=0.6, \Omega_{M}=0.4$.) The bottom-most curves are the signal strengths after one year of signal integration, for BH/BH binaries 10 years and 100 years before their merger. (Figure adapted from Flanagan and Hughes 40 .)

should easily be able to measure, while LIGO might not quite see it.

Unfortunately, it is far from obvious whether the event rate for such SMBH mergers will be interestingly high: By contrast with advanced LIGO IFOs, where Kalogera and others estimate tens to thousands of BH/BH mergers per year (Box 1), Haehnelt 6 and others estimate for LISA's SMBH 
mergers out to $z=1$ a rate in the range $10^{-1}-10^{2}$ per year. Even if the coalescence rate is only $0.1 / \mathrm{yr}$, then LISA should still see $\sim 3 \mathrm{BH} / \mathrm{BH}$ binaries with $3000 M_{\odot} \lesssim M \lesssim 10^{5} M_{\odot}$ that are $\sim 30$ years away from their final merger. These slowly inspiraling binaries should be visible, with one year of integration, out to a redshift $z \sim 1$ (bottom part of Fig. 5).

Because the SMBH-merger $\mathrm{S} / \mathrm{N}$ is so high at $z=1$, LISA could detect such mergers to redshifts $z \gg 1$. SMBH's are not expected to form in the universe at redshifts any larger than $z \sim 30$, and perhaps somewhat less than this. Figure f shows that the merger waves can be detected to distances well beyond $z \sim 30$ for masses in the range $M \sim 10^{4}$ to $\sim 10^{7} M_{\odot}$. (Below a few times $10^{5} M_{\odot}$ a crucial role in making this possible is played by the redshift itself, which lowers the waves' frequency, bringing them into LISA's band of good sensitivity; see Fig. 国.)

In the standard picture of bottom-up structure formation, large galaxies are formed by the merger of protogalaxies (which are themselves formed from the mergers of smaller protogalaxies). If the the merging protogalaxies contain $10^{5} M_{\odot}$ BH's at their centers, dynamical friction will bring the BH's to the common center, plausibly (though not assuredly) to the point where gravitational radiation takes over and drives the BH's to merger in less than a Hubble time. Based on this picture, Phinney estimates that the merger rate for $10^{5} M_{\odot}$ BH's would be $\sim 1500 /$ yr at $z \sim 7, \sim 500 /$ yr at $z \sim 10$, and $\sim 1 /$ yr at $z \sim 2080$ - rates comparable to those of advanced LIGO IFOs (Box 1). Thus LISA has the possibility to reach back and shed light on the earliest epochs of galaxy and SMBH formation

\subsection{Inspirals of $\sim 1-1000 M_{\odot}$ Compact Objects into Massive BH's}

LISA has the potential to observe the final inspiral waves from compact objects (WD's, NS's and BH's) of any mass $\mu \gtrsim 1 M_{\odot}$ spiraling into central bodies of mass $3 \times 10^{5} M_{\odot} \lesssim M \lesssim 3 \times 10^{7} M_{\odot}$ out to $3 \mathrm{Gpc}$. Figure 1 shows the example of a $10 M_{\odot}$ black hole spiraling into a $10^{6} M_{\odot} \mathrm{SMBH}$ at $1 \mathrm{Gpc}$ distance. The inspiral orbit and waves are strongly influenced by the SMBH spin. Two cases are shown 6: an inspiraling circular orbit around a non-spinning hole, and a prograde, circular, equatorial orbit around a near maximally spinning hole $(a / M=0.999$ in Kerr-metric language). For each case three dots are shown on the signal curve - from left to right the signal strength and frequency one year, one month, and one day before the small BH plunges into the horizon of the SMBH. Table 1 shows, at each of these times, the ratio of the orbital circumference to the SMBH horizon circumference, $\mathcal{C} / \mathcal{C}_{H}$, the number of GW cycles left until plunge, $N_{\text {cyc }}$, and the signal to noise in a

cutlerThorne: submitted to World Scientific on February 5, 2008 
Table 1. The waves from a $10 M_{\odot}$ in circular equatorial orbit around a $10^{6} M_{\odot} \mathrm{SMBH}$.

\begin{tabular}{ccrrr}
\hline \hline SMBH Spin & Time to Plunge & $\mathcal{C} / \mathcal{C}_{H}$ & $N_{\text {cyc }}$ & $S / N$ \\
\hline & & & & \\
0.999 & $1 \mathrm{yr}$ & 6.80 & 185,000 & 200 \\
0.999 & $1 \mathrm{mo}$ & 3.05 & 40,900 & 60 \\
0.999 & $1 \mathrm{day}$ & 1.30 & 2,320 & 5 \\
0 & $1 \mathrm{yr}$ & 9.46 & 85,000 & 60 \\
0 & $1 \mathrm{mo}$ & 7.14 & 9,650 & 70 \\
0 & $1 \mathrm{day}$ & 6.22 & 366 & 20 \\
\hline \hline
\end{tabular}

bandwidth equal to frequency (or, if that band extends beyond plunge, then the total signal to noise in the remainder of the waves), $S / N$.

The numbers in Table 1 are remarkable: The small BH spends its last year in the immediate vicinity of the SMBH horizon, emitting $\sim 100,000$ cycles of waves. These waves should_carry, encoded in themselves, high-accuracy information about the SMBH 98 :

Ryan 99 has shown that, when any small-mass, compact object spirals into a much more massive, compact central body, the inspiral waves will carry a "map" of the massive body's external spacetime geometry. Since that geometry is uniquely characterized by the values of the body's multiple moments, we can say equivalently that the inspiral waves carry, encoded in themselves, the values of all the body's multipole moments. By measuring the inspiral waveforms and extracting their map (i.e., measuring the lowest few multipole moments), we can determine whether the massive central body is a black hole or some other kind of exotic compact object 99 . The huge number of wave cycles, in the example in Table 11, implies the possibility of a high-precision map.

While the $\mathrm{S} / \mathrm{N}$ values in Table 1 are large enough (up to 200) to seem encouraging for detecting these waves and extracting the map that they carry, there are serious worries. These S/N's assume optimal signal processing (via matched filters), but it is highly unlikely that optimal processing can be achieved. The reasons are two:

First: Models of galactic nuclei, where massive holes (or other massive central bodies) reside, suggest that inspiraling stars and small holes typically will be in rather eccentric orbits 100.101. This is because they get injected into such orbits via gravitational deflections off other stars, and by the time gravitational radiation reaction becomes the dominant orbital driving force, there is not enough inspiral left to strongly circularize their orbits. Such orbital 
eccentricity will complicate the waveforms and complicate the extraction of information from them. In fact, the waveforms are likely to be so complex that the number of discrete templates needed in a in one-year coherent-integration search for these waves may be enormously larger than modern computers can handle. As a result, the signal processing may have to be done coherently over short stretches of time with incoherent techniques used to combine successive stretches, leading to a significant loss of $S / N$. Efforts to generate waveforms from generic orbits and then scope out this problem are barely getting started, as of winter 2001-02. (The waveforms are fairly well understood for generic orbits in the SMBH equatorial plane 102 and for circular orbits inclined to the equator, 103 but inclined generic orbits have not yet been studied, and the counting of templates even for equatorial orbits and inclined circular orbits has barely begun 104. Although the influence of radiation reaction on the orbital evolution is not yet fully understood 105, it is probably known well enough to scope out the data analysis problem.)

Second: There may be a significant loss of $S / N$ due to "source confusion" - i.e. due to the problem of separating out individual inspiral waveforms from the background due to other, weaker inspiral sources. At high frequencies this confusion noise could perhaps dominate all other noise sources, just as

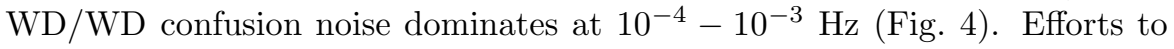
scope this out are also barely getting started.

The event rates for inspirals into SMBH's are not well known. Phinney 9 (relying in part on simulations of star-cluster evolution around a SMBH by Freitag 106 ) has shown that the detection rate is likely to be dominated by $\sim$ $10 M_{\odot}$ BH's spiraling into $\sim 10^{6} M_{\odot}$ SMBH's. (Phinney shows that $\sim 10^{6} M_{\odot}$ SMBH's are likely to have larger relaxed stellar cusps around them than either smaller or bigger BH's; coincidentally $10^{6} M_{\odot}$ is the optimum SMBH mass for LISA. And while inspirals of $\sim 10 M_{\odot}$ BH's probably have a smaller space density than inspirals of $\sim 1.4 M_{\odot}$ NS's, because of their larger $\mathrm{S} / \mathrm{N}$, they can be detected throughout a volume that is $(\sqrt{10 / 1.4})^{3} \sim 20$ times larger.)

The stimated inspiral rate for $\sim 10 M_{\odot}$ BH's is $\sim 1-10 /$ yr out to $1 \mathrm{Gpc} 97.601$, where the $S / N$ 's would be $\sim 60$ to $\sim 200$ if optimal signal processing were possible. If the loss of $S / N$ due to signal-processing problems is much larger than an order of magnitude (which it might be), then there could be difficulty detecting these inspirals. This motivates exploring a change of LISA's design so its noise curve is lowered by a factor 4 . Since such a change might significantly increase LISA's cost, it is greatly hoped that a scoping of the data analysis problem and a firming of the event rate will reveal that no design change is needed!

cutlerThorne: submitted to World Scientific on February 5, 2008 


\subsection{Collapse of Supermassive Stars}

New and Shapiro 107 have investigated the possibility of substantial GW emission from the collapse of supermassive stars (SMS's) to form SMBH's in galactic nuclei. The formation mechanism for SMBH's is still highly uncertain; one path could lead through rotating SMS's. While non-rotating SMS's suffer from a well-known radial collapse instability (arising from GR effects), even a small amount of rotation can stabilize the star 108. As the SMS cools and contracts, the ratio of $\beta=T /|W|$ (ratio of kinetic to gravitational binding energy) grows, and (for sufficiently large initial $\beta$ ) the star eventually becomes unstable either to mass-shedding at the equator or to a dynamical, $m=2$ (bar-mode) instability. New and Shapiro show that if viscosity and magnetic fields are insufficient to enforce uniform rotation (so that angular momentum is conserved on cylinders during the contraction), then the bar-mode instability is likely to occur first.

If the bar-mode perturbation grows to order unity and is long-lived, a substantial fraction of the star's rest mass-energy could be radiated in GW's. These waves end up in the LISA band for a range of plausible initial parameters. For example, for SMS mass $M \sim 10^{6} M_{\odot}$, initial (pre-collapse) radius $R \sim 10^{17} \mathrm{~cm}$ and initial $\beta \sim 10^{-6}$, New and Shapiro 107 estimate the SMS could radiate away $\sim 10^{3} M_{\odot}$ in GW's at $f \sim 3 \times 10^{-4} \mathrm{~Hz}$, in a "burst" lasting $\hat{\tau} \sim 1 \mathrm{yr}$, which is $n=f \hat{\tau} \sim 10^{4}$ cycles. If optimal signal processing could be used to search for these waves, the signal to noise ratio would be $S / N \simeq h_{c} / h_{n}$ where $h_{c} \equiv h \sqrt{n} \sim 10^{-18}$ at $1 \mathrm{Gpc}$ distance; here $h$ is the wave amplitude as estimated by New and Shapiro (corrected by them from a different value given in their paper), and $h_{c}$ is called the waves' characteristic amplitude 100 . Com-

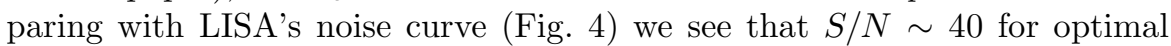
signal processing. With sufficiently accurate templates from numerical simulations of the collapse, it might be possible to achieve a search within a factor 4 of optimal, so $S / N \sim 10$, which would be adequate for confident detection; but as yet it is far from clear one can do so well - especially as the search must be performed in the thick of the WD-WD background. To get a clearer handle on the prospects will require considerable analysis - both source simulations and data-analysis explorations.

\subsection{LISA and the GW Stochastic Background}

While ground-based detectors can search for a stochastic background by crosscorrelating their outputs, this method is not available to LISA, since only one space-based detector is currently planned.

However, it is possible to combine the signals from the 3 spacecraft to

cutlerThorne: submitted to World Scientific on February 5, 2008 
construct a "symmetrized Sagnac observable", which is practically insensitive to both laser phase noise and gravitational waves 86 . With the aid of this observable, one can directly measure (symmetrized averages of) the proof-mass noise and optical-path noise, and thereby determine the instrumental noise power in the (sum of the) 3 Michelson channels used to detect GW's. Any excess "noise" above this power is due to GW's. This method is most effective in frequency bands where either the proof-mass noise or optical-path noise greatly dominates over the other. With this approach, in a one-year integration LISA could detect $h_{100}^{2} \Omega \gtrsim 10^{-11}$ in either the $10^{-4.5}<f<10^{-3.5} \mathrm{~Hz}$ or $10^{-2.8}<f<10^{-1.6} \mathrm{~Hz}$ bands 110 . Unfortunately, LISA's sensitivity to a GW background of early-universe origin is about an order-of-magnitude worse than this, since any primordial source with $\Omega(f) \lesssim 10^{-10}$ will be "covered up" by (the isotropic portion of) the galactic and extragalactic WD/WD binary backgrounds. LISA's low-frequency (LF) sensitivity of $\Omega \sim 10^{-10}$ is about two orders of magnitude better than the adjacent-band sensitivities of advanced LIGO IFOs $\left(\sim 5 \times 10^{-9}\right.$ in the HF band) and current pulsar timing $\left(\sim 10^{-8}\right.$ in the VLF band); Sec. 2.9.

In Sec. 2.9 we discussed a number of speculative early-universe sources that could produce GWs in earth-based detectors' HF band. Variants of all these sources could also radiate in LISA's LF band: (i) Universal inflation (with the standard model predicting waves much too weak for LISA to see, $\Omega \lesssim 10^{-16}-10^{-15}$, while some less plausible models such as the pre-bigbang suggest stronger waves); (ii) Phase transitions of quantum fields (LISA's frequency band is ideal for probing the electroweak phase transition, which should have occurred at universe age $\sim 10^{-12}$ sec and if strongly first order could have produced waves strong enough for LISA to see 111); (iii) Goldstone modes of scalar fields that arise in supersymmetric and string theories; (iv) excitations of our universe as a brane in higher dimensions (with LISA probing excitation lengthscales $\sim 1-10^{-5} \mathrm{~mm}$ if there are one or two relevant higher dimensions); and (v) Cosmic strings (with LISA's searches for bursts from string cusps and kinks able to constrain string tensions to $\mu \lesssim 10^{-13}$ ). For further discussion and references, see Sec. 2.9.

There is some hope that a follow-on mission to LISA might reach the sensitivity required for probing standard inflation, $\Omega(f) \sim 10^{-16}-10^{-15}$. To do so will require that the mission's detectors operate outside the regime dominated by close binaries. A "short-LISA" with $\sim 100$ times shorter arms would have good sensitivity at $f \sim 1 \mathrm{~Hz}$, where the WD binaries disappear and the binary background is dominated by NS binaries; a constellation of 3 short-LISA's spaced evenly around the Sun (along Earth's orbit) could have angular resolution $\Delta \theta \sim 10^{-3} \mathrm{rad}$, allowing (probably) the NS binaries to be 
detected individually and subtracted out. At present, such a constellation of $\sim 9-12$ satellites seems the best possibility for probing the inflationary GW background from space.

\section{Conclusions}

It is now about 40 years since Joseph Weber initiated his pioneering development of gravitational-wave detectors 112 , and 30 years since Robert Forward 113 and Rainer Weiss 114 initiated work on interferometric detectors. Since then, hundreds of talented experimental physicists have struggled to improve the sensitivities of gravitational-wave detectors, and hundreds of theorists have explored general relativity's predictions.

These two parallel efforts are now intimately intertwined and are pushing toward an era in the not distant future, when measured gravitational waveforms will be compared with theoretical predictions to learn how many and what kinds of relativistic objects really populate our Universe, and how these relativistic objects really are structured and really behave when quiescent, when vibrating, and when colliding. By about the time of GR-18, if not sooner, we should have some answers.

\section{Acknowledgments}

Much of this manuscript's Section 2 (high-frequency waves) was written in close consultation with Lars Bildsten, Alessandra Buonanno, Craig Hogan, Vassiliki Kalogera, Benjamin J. Owen, E. Sterl Phinney, Thomas A. Prince, Frederic A. Rasio, Stuart L. Shapiro, Kenneth A. Strain, Greg Ushomirsky, and Robert V. Wagoner. We warmly thank them for their significant contributions to this review. We also thank Sam Finn for especially valuable input into our sections on supernovae, pulsars, and gamma ray bursts. Thorne's contributions to this review were supported in part by NSF grant PHY-0099568 and NASA grant NAG5-10707. Cutler's work was partly supported by NASA grant NAG5-4093.

\section{References}

1. E. E. Flanagan. In Naresh Dadhich and Jayant Narlikar, editors, Gravitation and Relativity: At the turn of the Millennium, 15th International Conference on General Relativity and Gravitation. Inter-University Centre for Astronomy and Astrophysics, 1998.

cutlerThorne: submitted to World Scientific on February 5, 2008 
2. Lee Samuel Finn. In Joan M. Centrella, editor, Astrophysical Sources for Ground-based Gravitational Wave Detectors, volume 575 of AIP Conference Proceedings, Melville, New York, 2001. American Institute of Physics. gr-qc/0104042.

3. S. Hughes, S. Marka, P.L. Bender, and C.J. Hogan. In Proceedings of the 2001 Snowmass Meeting. astro-ph/0110349.

4. K.S. Thorne. In R.M. Wald, editor, Black Holes and Relativistic Stars, page 41. University of Chicago Press, 1998.

5. K.S. Thorne. The scientific case for advanced ligo interferometers. 2001. LIGO Document Number P-000024-00-R; available from LIGO Document Control Center, http://admdbsrv.ligo.caltech.edu/dcc/.

6. M. Kamionkowski and A. Jaffe. Int. J. Mod. Phys. A, 16:116, 2001.

7. A.N. Lommen and D.C. Backer. Astrophys. J., 2002. in press; astro$\mathrm{ph} / 0107470$.

8. S. Kawamura. 2002. article in this volume.

9. B.F. Schutz. 2002. article in this volume.

10. N.A. Robertson. Class. Quant. Grav., 17:R19, 2000.

11. B. Barish and R. Weiss. Physics Today, 52(10):44, 2001.

12. M. Tinto and J.W. Armstrong. Phys. Rev. D, 58:042002, 1998.

13. See http://igec.lnl.infn.it/.

14. This $\tilde{h}_{s}(f)$ is related to the characteristic amplitude $h_{c}(f)$ widely used in the literature in the following way: $h_{c}=\sqrt{5 f}(T / N) \tilde{h}_{s}$, where the $\sqrt{5}$ comes from averaging over the sky, the $\sqrt{f}$ is due to $h_{c}$ being the signal strength in a bandwidth equal to frequency, and $T / N$ is the ratio of the threshold to the rms noise at the endpoint of signal processing.

15. If, in its advanced ("LIGO-II") incarnation, the $2 \mathrm{~km}$ interferometer is replaced by a narrow-banded $4 \mathrm{~km}$ interferometer, then for the most important wide-band sources, inspiraling binaries, the 3-IFO threshold will be lowered (improved) slightly and correspondingly the distances to which the sources can be seen will be increased slightly beyond those in Box 1. If the $2 \mathrm{~km}$ interferometer is replaced by a wide-band $4 \mathrm{~km}$ interferometer, then the threshold will be lowered by a factor 1.16, the binaries' observable distances will be increased correspondingly by 1.16 , and the predicted event rates will go up by $1.16^{3} \simeq 1.5$.

16. See, e.g., B.J. Owen and B.S. Sathyaprakash. Phys. Rev. D, 60:022002, 1999. and references therein.

17. L. Bildsten. Astrophys. J. Lett., 501:L89, 1998.

18. G. Ushomirsky, C. Cutler, and L. Bildsten. Mon. Not. Roy. Astron. Soc., 319:902, 2000.

19. M. van der Klis. Ann. Rev. Astron. Astrophys., 38:717, 2000. 
20. P.R. Brady and T. Creighton. Phys. Rev. D, 61:082001, 2000.

21. M.A. Papa, B.F. Schutz, and A.M. Sintes. In Proceedings of ICTP Conf. on Gravitational waves: a challenge to theoretical astrophysics. gr-qc/0011034.

22. P.R. Brady, T. Creighton, C. Cutler, and B.F. Schutz. Phys. Rev. D, 57:2101, 1998 .

23. B. Allen and J.D. Romano. Phys. Rev. D, 59:102001, 1999.

24. See the discussion of the overlap reduction function in Sec. III.B of Allen and Romano (1999), especially Fig. 2.

25. The NS/NS numbers in this table are taken from V. Kalogera, R. Narayan, D. Spergel, and J.H. Taylor, Astrophys. J., 556:340, 2001 - as augmented slightly by a new channel for NS/NS formation analyzed by K. Belczynski and V. Kalogera, Astrophys. J. Lett., 550:L183, 2001 - and with adjustment of $\mathcal{D}_{\mathrm{WB}}$ from $350 \mathrm{Mpc}$ (appropriate for three $4 \mathrm{~km}$ interferometers) to $300 \mathrm{Mpc}$ (for one $2 \mathrm{~km}$ and two $4 \mathrm{~km}$ interferometers 15 ). The NS/BH and BH/BH numbers are based on Kalogera's survey of all estimates of $\mathcal{R}_{\text {gal }}$ [V. Kalogera, private communication winter 2000-01; V. Kalogera in Gravitational Waves, Proceedings of the Third Edoardo Amaldi Conference, AIP Conference Proceedings Vol. 523, ed. Sydney Meshkov (American Institute of Physics, 2000), astro-ph/9911532; and V. Kalogera in Astrophysical Sources of Gravitational Radiation for Ground-Based Detectors, ed. J.M. Centrella (AIP, Woodbury NY), astroph/0101047] — extrapolated into the distant universe by the B-band method of Phinney 26 as updated by Kalogera et. al. The last column is based on the estimates by McMillan and Portegies Zwart 30. The numbers preceded by " $\lessgtr$ " represent Kalogera's personal best estimate of the lower limit with the "<" being a warning that some researchers have argued for lower numbers.

26. E.S. Phinney. Astrophys. J., 380:L17, 1991.

27. P. Madau. Physica Scripta, T85:156, 2000. astro-ph/9902228.

28. V. Kalogera. Astrophys. J., 541:319, 2000.

29. When extrapolating out to cosmological distances, $z \gtrsim 0.1$, we must take account of cosmological effects. We assume a cosmological model with $H_{o}=65 \mathrm{~km} / \mathrm{s}-\mathrm{Mpc}, \Omega_{M}=0.4$, and we take account of the evolution of the numbers of massive stars and thence $\mathrm{BH} / \mathrm{BH}$ binaries using Eq. (4) of Madau, Ref. 27; the result is an event rate, inside redshift $z$, that scales as $z^{3}$ for $0<z<4$, aside from an enhancement by a factor $\sim 2$ near $z=1$ and a decrement by a factor $\sim 2$ at $z \sim 2.5-4$.

30. S. McMillan and S. Portegies Zwart. Astrophys. J. Lett., 528:L17, 2000.

31. C. Cutler and E. E. Flanagan. Phys. Rev. D, 49:2658, 1994.

cutlerThorne: submitted to World Scientific on February 5, 2008 
32. E. Poisson and C. M. Will. Phys. Rev. D, 52:848, 1995.

33. T. A. Apostolatos, C. Cutler, G. J. Sussman, and K. S. Thorne. Phys. Rev. D, 49:6274, 1994.

34. L. E. Kidder. Phys. Rev. D, 52:821, 1995.

35. T. A. Apostolatos. Phys. Rev. D, 52:605, 1995.

36. P. Jaranowski, K.D. Kokkotas, A. Krolak, and G. Tsegas. Class. Quant. Grav., 13:1279, 1996.

37. E. Poisson. Phys. Rev. D, 47:1497, 1993.

38. M. Vallisneri. Phys. Rev. Lett., 84:3519, 2000.

39. J.A. Faber and F.A. Rasio. Phys. Rev. D. in press; gr-qc/0201040.

40. E. E. Flanagan and S. A. Hughes. Phys. Rev. D, 57:4535, 1998.

41. E. E. Flanagan and S. A. Hughes. Phys. Rev. D, 57:4566, 1998.

42. A. Burrows, J. Hayes, and B. Fryxell. Astrophys. J., 450:830, 1995.

43. http://online.itp.ucsb.edu/online/neustars00/chernoff/, especially slide 34.

44. K. Nomoto, in Proc. 13th Texas Symposium on Relativistic Astrophysics, ed. M. Ulmer (World Scientific: Singapore); K. Nomoto and Y. Kondo, Astrophys. J. Lett., 367:L19, 1991.

45. See, e.g., recent simulations of dynamical bar formation by M. Shibata, T.W. Baumgarte and S.L. Shapiro, Astrophys. J. 542:453, 2000, and M. Saijo, J. Shibata, T.W. Baumgarte and S.L. Shapiro, Astrophys. J., 548:919, 2001, and references therein. These simulations show that general relativity enhances the instability to bar formation.

46. K.C.B. New, J.M. Centrella, and J.E. Tohline. Phys. Rev. D, 62:064019, 2000.

47. D. Brown. Phys. Rev. D, 62:084024, 2000.

48. D. Lai and S.L. Shapiro. Astrophys. J., 442:259, 1995.

49. In order to shrink to normal neutron-star size, the proto-neutron-star must get rid of its excess angular momentum. In the idealized case that (i) the angular momentum is lost solely to gravitational waves and not to hydrodynamic or magnetic processes, and (ii) the LIGO data are analyzed by optimal signal processing, the integrated signal strength depends primarily on the excess angular momentum and not on the ellipticity of the bar, and the distance to which the signal can be seen is of order $150 \mathrm{Mpc}$ (about half that for a NS/NS binary whose mass is twice as large). A factor ten reduction of amplitude signal to noise (factor 100 reduction of power signal to noise), due to non-optimal signal processing and to loss of angular momentum via non-gravitational-wave channels, would still leave the source detectable at $\sim 15 \mathrm{Mpc}$, the distance of Virg

50. L.S. Finn. Section titled "Core-Collapse Supernovae" in Ref. 2, and pri-

cutlerThorne: submitted to World Scientific on February 5, 2008 
vate communication about further details.

51. C. Cutler and D.I. Jones. Phys. Rev. D, 63:024002, 2001.

52. C. Cutler, G. Ushomirsky, and B. Link. in preparation.

53. M. Zimmermann and E. Szedenits. Phys. Rev. D, 20:351, 1979.

54. S. L. Shapiro and S. A. Teukolsky. Wiley: Interscience, 1983. Section 10.10 and references cited therein.

55. S. Chandrasekhar. Phys. Rev. Lett., 24:611, 1970.

56. J. L. Friedman and B. F. Schutz. Astrophys. J., 222:281, 1978.

57. R. V. Wagoner. Astrophys. J., 278:345, 1984.

58. L. Lindblom. Astrophys. J., 438:265, 1995.

59. L. Lindblom and G. Mendell. Astrophys. J., 444:804, 1995.

60. L. Lindblom. Astrophys. J., 438:265, 1995.

61. N. Andersson. Astrophys. J., 502:708, 1998.

62. J. Friedman and S. Morsink. Astrophys. J., 502:714, 1998.

63. L. Lindblom, B.J. Owen, and S. Morsink. Phys. Rev. Lett., 80:4843, 1998.

64. N. Andersson, K. Kokkotas, and B. Schutz. Astrophys. J., 510:846, 1999.

65. L. Rezzolla, F.L. Lamb, D. Markovic, and S.L. Shapiro. Phys. Rev. D, 64:104014, 2001.

66. L. Bildsten and G. Ushomirsky. Astrophys. J., 529:L33, 2000.

67. P.B. Jones. Phys. Rev. D, 64:084003, 2001.

68. L. Lindblom and B.J. Owen. Submitted; astro-ph/0110558.

69. P. Arras, E.E. Flanagan, S.M. Morsink, A.K. Schenk, S.A. Teukolsky, and I. Wasserman. Astrophys. J.., 2002. Submitted; astro-ph/0202345.

70. See, e.g., P. Meszaros, M.J. Rees and R.A.M.J. Wijers, New Astron., 4:303, 1999.

71. M. Schmidt. Astrophys. J., 559:L79, 2001.

72. C.S. Kochanek and T. Piran. Astrophys. J., 417:L17, 1993.

73. M.H.P.M. van Putten. Physics Reports, 345:1, 2001.

74. L.S. Finn, S.D. Mohanty, and J. Romano. Phys. Rev. D, 60:121101, 1999.

75. M.S. Turner. Phys. Rev. D, 55:435, 1997.

76. L. P. Grishchuk. Soviet Physics-JETP, 40:409, 1974.

77. R. Brustein, M. Gasperini, M. Giovannini, and G. Veneziano. Phys. Lett. $B, 361: 45,1995$. For a review of more recent literature, see M. Maggiore. Phys. Rep., 331:283, 2000.

78. M. Kamionkowski, A. Kosowsky, and M.S. Turner. Phys. Rev. D, 49:2837, 1994.

79. See, e.g., C.J. Hogan, Phys. Rev. Lett 74, 3105 (1995), and C.J. Hogan, astro-ph/9809364. 
80. C.J. Hogan. Phys. Rev. Lett., 85:2044, 2000.

81. C.J. Hogan. Phys. Rev. D, 62:121302, 2000.

82. B. Allen and J.D. Romano. Phys. Rev. D, 59:102001, 1999.

83. T. Damour and A. Vilenkin. Phys. Rev. D., 64:064008, 2001.

84. B. Allen. In J. A. Miralles, J. A. Morales, and D. Saez, editors, Proceedings of the Spanish Relativity Meeting, '96, page 3. 1996. gr-qc/9604033.

85. P. Bender et. al. LISA, Laser interferometer space antenna for the detection and observation of gravitational waves: Pre-Phase A Report. Max-Planck-Institut für Quantenoptik, MPQ 208, December 1995.

86. J.W. Armstrong, F.B. Estabrook, and M. Tinto. Class. Quant. Grav., 18:4059, 2001.

87. L. S. Finn and K. S. Thorne. Phys. Rev. D, 62:124021, 2000.

88. E.S. Phinney. Lisa science requirements. 2001. Presentation to LISA International Science Team, 12 December 2001; available at LIST Working Group 1 Website, http://www.tapir.caltech.edu/listwg1/.

89. C. Ungarelli and A. Vecchio. Phys. Rev. D, 64:121501, 2001.

90. C. Cutler and A. Vecchio. In W.M. Folkner, editor, Proceedings of the 2nd International LISA Symposium, page 95. American Institute of Physics, 1998.

91. S. Hughes, private communication.

92. A. Vecchio. In S. Meshkov, editor, Proceedings of the Third Amaldi Conference on Gravitation al Waves, page 238. American Institute of Physics, 2000. gr-qc/9912099.

93. D. Christodoulou. Phys. Rev. Lett., 67:1486, 1991.

94. K. S. Thorne. Phys. Rev. D, 45:520-524, 1992.

95. A. G. Wiseman and C. M. Will. Phys. Rev. D, 44:R2945, 1991.

96. M. G. Haehnelt. Mon. Not. Roy. Astron. Soc., 269:199, 1994.

97. E.S. Phinney, private communication.

98. F. D. Ryan. Phys. Rev. D, 56:1845, 1997.

99. F. D. Ryan. Phys. Rev. D, 52:5707, 1995.

100. D. Hils and P. Bender. Astrophys. J. Lett, 445:L7, 1995.

101. S. Sigurdsson and M. J. Rees. Mon. Not. Roy. Astron. Soc., 284:318, 1997.

102. C. Glampedakis and D. Kennefick. 2002. In preparation.

103. S.A. Hughes. Phys. Rev. D, 64:064004, 2001. And references therein.

104. S. Hughes. LIST Working Group 1 Website, http://www.tapir.caltech.edu/listw g1/.

105. L. Barack, Y. Mino, H. Nakano, A. Ori, and M. Sasaki. Phys. Rev. Lett., 88:091101, 2002. And references therein.

106. M. Freitag. Class. Quant. Grav., 18:4033, 2001.

cutlerThorne: submitted to World Scientific on February 5, 2008 
107. K.C.B. New and S.L. Shapiro. Astrophys. J., 548:439, 2001. And private communication.

108. W.A. Fowler. Astrophys. J., 184:180, 1966.

109. K. S. Thorne. In S. W. Hawking and W. Israel, editors, Three Hundred Years of Gravitation, pages 330-458. Cambridge University Press, 1987.

110. C.J. Hogan and P.L. Bender. Phys. Rev. D, 64:062002, 2001.

111. A. Kosowsky and M. S. Turner. Phys. Rev. D, 49:2837, 1994.

112. J. Weber. Phys. Rev., 117:306, 1960.

113. G. E. Moss, L. R. Miller, and R. L. Forward. Applied Optics, 10:2495, 1971.

114. R. Weiss. Quarterly Progress Report of RLE, MIT, 105:54, 1972. 